\title{
The Recent Progress on Halide Perovskite-Based Self-Powered Sensors Enabled by Piezoelectric and Triboelectric Effects
}

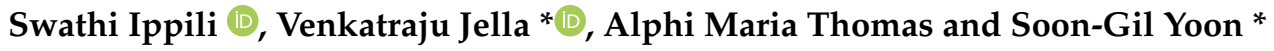 \\ Department of Materials Science and Engineering, Chungnam National University, Daeduk Science Town, \\ Daejeon 34134, Korea; iswathirs@gmail.com (S.I.); alphirosary@gmail.com (A.M.T.) \\ * Correspondence: venkatrajujella@gmail.com (V.J.); sgyoon@cnu.ac.kr (S.-G.Y.)
}

Citation: Ippili, S.; Jella, V.; Thomas, A.M.; Yoon, S.-G. The Recent Progress on Halide Perovskite-Based Self-Powered Sensors Enabled by Piezoelectric and Triboelectric Effects. Nanoenergy Adv. 2021, 1, 3-31. https://doi.org/10.3390/ nanoenergyadv1010002

Academic Editor: Ya Yang

Received: 28 May 2021

Accepted: 15 July 2021

Published: 23 July 2021

Publisher's Note: MDPI stays neutral with regard to jurisdictional claims in published maps and institutional affiliations.

Copyright: () 2021 by the authors. Licensee MDPI, Basel, Switzerland. This article is an open access article distributed under the terms and conditions of the Creative Commons Attribution (CC BY) license (https:// creativecommons.org/licenses/by/ $4.0 /)$.

\begin{abstract}
Sensors have recently gathered significant attention owing to the rapid growth of the Internet of Things (IoT) technology for the real-time monitoring of surroundings and human activities. Particularly, recently discovered nanogenerator-based self-powered sensors are potential candidates to overcome the existing problems of the conventional sensors, including regular monitoring, lifetime of a power unit, and portability. Halide perovskites (HPs), with an excellent photoactive nature, dielectric, piezoelectric, ferroelectric, and pyroelectric properties, have been potential candidates for obtaining flexible and self-powered sensors including light, pressure, and temperature. Additionally, the photo-stimulated dielectric, piezoelectric, and triboelectric properties of HPs make them efficient entrants for developing bimodal and multimode sensors to sense multi-physical signals individually or simultaneously. Therefore, we provide an update on the recent progress in self-powered sensors based on pyroelectric, piezoelectric, and triboelectric effects of HP materials. First, the detailed working mechanism of HP-based piezoelectric, triboelectric, and pyroelectric nanogeneratorsoperated as self-powered sensors-is presented. Additionally, the effect of light on piezoelectric and triboelectric effects of HPs, which is indispensable in multimode sensor application, is also systematically discussed. Furthermore, the recent advances in nanogenerator-based self-powered bimodal sensors comprising HPs as light-active materials are summarized. Finally, the perspectives and continuing challenges of HP-based self-powered sensors are presented with some opportunities for future development in self-powered multimode sensors.
\end{abstract}

Keywords: halide perovskite; nanogenerator; PENG; PyENG; TENG; self-powered sensor; temperature sensor; pressure sensor; physiological sensor; photodetector

\section{Introduction}

The wireless networks and devices enable the new technology to collect and share information through wireless communication systems owing to the rapid development of the Internet of Things (IoT) and artificial intelligence (AI) technology [1]. Sensors are the key components in these systems, which can measure and quantify any external physical features from the various external stimuli, such as light, pressure, temperature, chemical, and biological property [2-6]. However, most of the sensors are powered by external power sources, such as batteries. Their usage in sensors is faced with several issues, including regular monitoring, frequent charging process, limited life cycles, high maintenance cost, and environmental pollution. Moreover, they also obstruct the construction of flexible sensors due to their rigid nature $[7,8]$. Therefore, the development of maintenance-free, self-powered sensors that cannot rely on a conventional power source is highly necessary to be implemented in next-generation portable (wearable) electronics, IoTs, and smart cities [9-11]. In recent years, the self-powered operation of such sensors has been achieved using nanogenerators, in which the harvester converts the ambient energy into electrical energy and power the sensing unit continuously $[9,12]$. Nanogenerators can be categorized primarily into the following three types based on the working mechanism 
to generate electricity: piezoelectric nanogenerator (PENG), triboelectric nanogenerator (TENG), and pyroelectric generator (PyENG). Nanogenerators have been extensively used as harvesters - to harvest the available energy from the surrounding environment-and used as sensors simultaneously $[13,14]$. Pressure or strain sensors are widely used in health monitoring, motion detection, robotics, electronic skin, and human-machine interaction [15-20]. Nanogenerator-based pressure sensors are used in dynamic pressure sensing applications owing to their simple fabrication process, low cost, and easy electrical signal acquisition [21,22]. Moreover, pyroelectric materials can be used to harvest the thermal energy to be converted into electrical energy owing to the generation of an internal polarization in response to changes in temperature $[23,24]$. In the past decades, numerous materials, including ceramics (e.g., $\mathrm{BaTiO}_{3}, \mathrm{PZT}, \mathrm{NaNbO}_{3}$ ), semiconductors (e.g., $\mathrm{ZnO}$, GaN, CdS), and polymers (e.g., PVDF and its copolymers) have been investigated in detail to fabricate nanogenerators and sensors [25-31]. Among them, ceramic perovskite materials are widely used to obtain high-performance and efficient nanogenerators and sensors owing to their remarkable dielectric, ferroelectric, piezoelectric, and pyroelectric properties [32-34]. However, the high-temperature syntheses, calcination process, as well as the brittle and rigid nature of these materials limit their applicability in flexible devices. Therefore, novel materials with high ferroelectric/piezoelectric properties that can be produced via cost-effective and low-temperature solution processes are highly desirable.

Halide perovskites (HPs), including both organic-inorganic and inorganic HPs, have received widespread attention for photovoltaic research. They have obtained high-efficiency solar cells with the power conversion efficiency (PCE) of over $25 \%$ because of their simple synthesis processes and impressive characteristics such as high absorption coefficients, band gap tunability, long-carrier diffusion lengths, and long-carrier lifetimes [35-39]. Additionally, HPs are used to develop various devices, such as a photodetectors, light-emitting diodes, lasers, nanogenerators, and thin-film transistors [40-43]. The nanogenerators, capable of producing electricity from ambient unused energy sources, including thermal gradient and mechanical vibrations, have been recent use of HPs due to their impressive dielectric and piezoelectric properties [44,45]. Numerous flexible nanogenerators, including PENG, TENG, and PyENG, have been constructed using HP and their polymer composite materials by compositing with various piezoelectric polymers (e.g., poly (vinylidene fluoride)) and non-piezoelectric polymers (e.g., polydimethylsiloxane (PDMS)) to demonstrate high-performance owing to their mechanical flexibility $[44,46,47]$. These HP-based nanogenerators are highly sensitive to various external stimuli, such as pressure, temperature, and light. Temperature fluctuations and pressure in the nanogenerator can cause the dipole oscillation in active HP materials, leading to the potential difference across the device; it would produce electricity [44,48]. Similarly, contact electrification between two dissimilar triboelectric materials generates the electric signals [49]. Hence, controlling the performance of nanogenerators under external perturbations would predominantly enable them to act as a self-powered pressure or temperature sensor without relying on a battery [48,50]. Moreover, illuminating nanogenerator significantly alters their output performance owing to the combined properties of piezoelectric or triboelectric and optoelectronic properties [51,52]. Such light-stimulated performance is highly feasible to detect light or quantity of light intensity without depending on an external power source [53,54]. Additionally, HPs have diverged towards bimodal/multimode physical signals_light and pressure-sensing applications owing to their combined light-absorbing property and piezoelectric/triboelectric effect [51,55].

We provide an update on the recent progress of self-powered sensors based on nanogenerators, which were fabricated using HP materials and their polymer composites. First, the detailed working mechanisms of PENG, TENG, and PyENG that can operate as self-powered sensors are discussed. Additionally, the operation of the PENG and TENG as self-powered photodetectors under illumination is also discussed with the possible underlying mechanisms. Second, we systematically review HP-based nanogenerators such as temperature, pressure, physiological sensor, and photodetectors to sense various 
external factors, such as pressure, temperature, and light. Third, an overview of the HPbased bimodal sensors for simultaneous sensing of pressure and light signals is presented. Furthermore, we suggest the future projections of the research towards developing singlestructure multimodal sensing devices. Finally, we briefly present perspectives, current challenges, and future prospects of HP-based self-powered sensors.

\section{Discussion}

\subsection{Halide Perovskites (HPS)}

Halide perovskite materials consist of three-dimensional $\mathrm{ABX}_{3}$-type crystal structures (Figure 1, crystal structure). In $\mathrm{ABX}_{3}$, A denotes the monovalent organic or inorganic cation (such as $\mathrm{MA}^{+}=\mathrm{CH}_{3} \mathrm{NH}_{3}{ }^{+}, \mathrm{FA}=\mathrm{CH}\left(\mathrm{NH}_{2}\right)_{2}{ }^{+}$and $\mathrm{Cs}^{+}$), $\mathrm{B}$ represents the divalent cation (such as $\mathrm{Pb}^{2+}, \mathrm{Sn}^{2+}$, and $\mathrm{Ge}^{2+}$ ), and $\mathrm{X}$ is the halide anion $(\mathrm{Cl}, \mathrm{Br}, \mathrm{I})$. In the crystal structure, cation $\mathrm{A}$ is coordinated with 12 neighboring $\mathrm{X}$, and cation $\mathrm{B}$ is connected with $6 \mathrm{X}$ anions, forming cuboctahedral and octahedral geometries, respectively. The structural formability of the $3 \mathrm{D}-\mathrm{ABX}_{3} \mathrm{HPs}$ and their stability were determined by the Goldschmidt tolerance factor $(t)$ and the octahedral factors $(\mu)[56,57]$. The tolerance factor, $t=\left(r_{A}+r_{X}\right) / \sqrt{ } 2\left(r_{B}+r_{X}\right)$, where $r_{A}, r_{B}$, and $r_{X}$ represent the effective ionic radii of $A, B$, and $X$ in the HP, respectively. The $r_{B} / r_{X}$ is defined as an octahedral factor $(\mu)$ and is directly correlated with a BX ${ }_{6}$ octahedron, playing an important role in multifunctional properties of HP materials. The tolerance factor values for HP materials were found to be within the 0.8-1.11 range, while the octahe$\mathrm{dral}$ factor values were in the range of $0.44-0.90[58,59]$. The calculated $t$ values for cubic, orthorhombic, and hexagonal structured HPs were $0.8<\mathrm{t}<1.0, \mathrm{t}<0.8$, and $\mathrm{t}>1$, respectively $[57,60]$. Numerous methods have been proposed to fabricate HP thin films, such as the one-step solution process, two-step sequential deposition, vapor deposition method, and vapor-assisted solution process [61-64]. Solution methods have been promoted due to simple and low-temperature fabrication ways and their compliance with flexible and large-scale devices $[36,44]$. Furthermore, the solution methods are promising to control crystalline formation better, leading to tunable properties at nano/micro-scale $[37,65]$. These HP-based materials have been considered to fabricate flexible nanogenerators owing to their impressive dielectric, ferroelectric, piezoelectric, and pyroelectric properties and beneficial features such as low-temperature and large-scale fabrication routes and controllable properties [66-70]. In particular, the dielectric and piezoelectric properties of HP-based materials are remarkable and nearly similar to those of ceramic materials $[44,65,66,71-73]$. Generally, the dielectric material is electrically an insulator and will readily polarize under an applied electric field. The ability of a material to store the electrical energy can be estimated by its dielectric permittivity $\left(\varepsilon_{\mathrm{r}}\right)$ values. However, the dielectric loss factor describes the inefficiency of a material to hold the stored energy. Consequently, material with high loss factor cannot hold the charge completely indicating leaky nature of sample. Therefore, materials with larger dielectric constant values and low dissipation factor values tend to generate higher output performance in nanogenerator applications because the nanogenerators are designed as part of capacitors [72]. Many researchers have demonstrated the dielectric properties of HP-based materials and also systematically examined the changes in dielectric properties of compositionally tuned HP materials $[44,65,66,73]$. The dielectric polarization mechanism in HP-based materials is nearly similar to well-known ceramic perovskite materials. The existing organic cation at the center of an HP-crystal structure (e.x., $\mathrm{MA}^{+}$cation in $\mathrm{MAPbI}_{3}$ ) disorders under an applied filed; thus, resulting in a dipolar polarization in HPs [44]. As depicted in Figure 2a, the most widely used $\mathrm{MAPbI}_{3}$ perovskite revealed an $\varepsilon_{\mathrm{r}}$ value of $\sim 52$ and low dissipation value of $\sim 0.02$ at an applied frequency of $100 \mathrm{kHz}$ [71]. Moreover, $\mathrm{FAPbI}_{3}$ perovskite exhibited an improved dielectric property due to presence of larger cation $\left(\mathrm{FA}^{+}\right)$that has larger dipole momentum compared to that of $\mathrm{MA}^{+}$cation [74]. In addition, the dielectric properties of HPs can be significantly enhanced by partial tuning of its composition. For example, the reported halide-doped $\mathrm{MAPbI}_{3}$ perovskite samples displayed a notable enhancement in dielectric properties upon halide doping (either $\mathrm{Cl}$ or $\mathrm{Br}$ ) [66]. In particular $\mathrm{MAPbI}_{3-\mathrm{x}} \mathrm{Cl}_{\mathrm{x}}$ displayed a 
high $\varepsilon_{\mathrm{r}}$ value of $\sim 90.9$. The improved property is not only because of improved grain size but also the existence of binary systems such as $\mathrm{MAPbI}_{3}$ and $\mathrm{MAPbCl}_{3}$ in the final film which led to interfacial polarizations between two phases. Similarly, the Fe-incorporated $\mathrm{MAPbI}_{3}$ unveiled enhanced $\varepsilon_{\mathrm{r}}$ value of $\sim 107$ upon partial incorporation of Fe $(\mathrm{x}=0.07)$ [65]. The improved dielectric properties promoted the enhancement in piezoelectric output performance of the $\mathrm{MAPb}_{1-\mathrm{x}} \mathrm{Fe}_{\mathrm{x}} \mathrm{I}_{3}$ based PENG. Furthermore, the lead-free Sn-based HP materials also possess high dielectric properties [45].

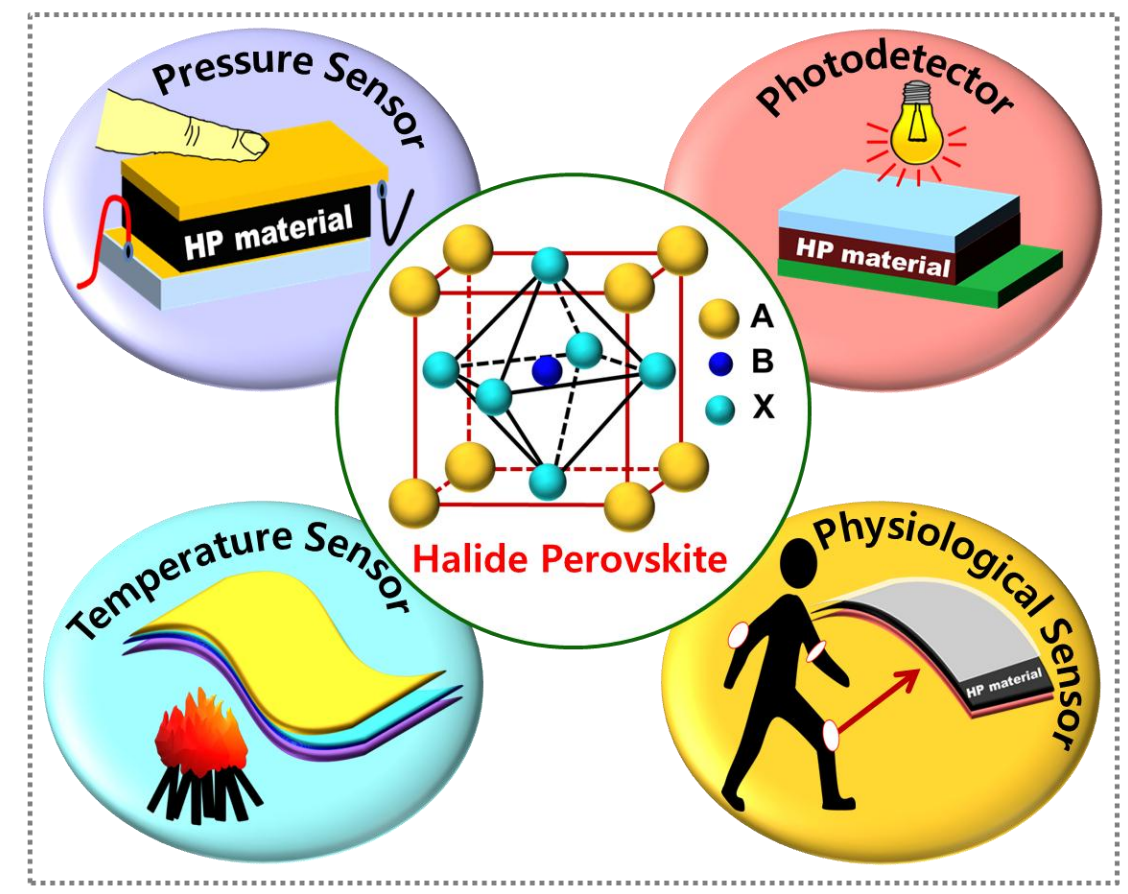

Figure 1. Schematic illustration of $\mathrm{ABX}_{3}$-type halide perovskite structure and their utility in nanogenerator based self-powered sensing applications.

In addition, the dielectric HPs were used as filler materials in polymer materials and observed the increment in dielectric properties of polymer materials with lower HP loading contents $[44,45,51,68]$. For example, the reported 25 vol $\% \mathrm{MAPbI}_{3}-\mathrm{PVDF}$ composite film showed much larger $\varepsilon_{\mathrm{r}}$ value of $\sim 56$ than the neat PVDF film $\left(\varepsilon_{\mathrm{r}} \sim 12.6\right)$ measured at $1 \mathrm{kHz}$ frequency [68]. Similarly, the $15 \mathrm{wt} \% \mathrm{MASnBr}_{3}-\mathrm{PDMS}$ composite sample also displayed a higher $\varepsilon_{\mathrm{r}}$ value of $\sim 36.23$, which is nearly ten times larger than only PDMS $\left(\varepsilon_{\mathrm{r}} \sim 3.38\right)$ film [45]. The rapid augmentation in dielectric properties of HP-polymer composites is predominantly because of existence of Maxwell-Wagner-Sillars (MWS) or interfacial polarizations between those two heterogeneous materials interfaces $[44,45,51,68]$. Furthermore, in case of HP-PVDF composites with increasing the content of HP material in PVDF matrix, the orientation of $\beta$-phase of PVDF will be notably improved due to enormous interactions (hydrogen bonding) between polar groups of HP material and PVDF material, which could also be another important cause for improved dielectric properties in composite films [68]. The conferred dielectric properties of HP/HP-polymer materials so far are investigated in dark state conditions. However, HP materials are promising light-active materials with excellent optoelectronic properties, which may be deliberated for light-dependent effects in dielectric properties of HPs. However, there have not been many reports elucidated the light effect on dielectric and piezoelectric properties of HP materials. J. Bisquert et al. have evidenced for the light-induced giant dielectric properties in $\mathrm{MAPbI}_{3-\mathrm{x}} \mathrm{Cl}_{\mathrm{x}}$ films in particularly at low frequency regions upon illumination [75]. The observed giant dielectric behavior of the sample was probably due to the rapid fluctuations in the perovskite unit cell and change in polarization emerged by the orientations of polar organic molecule $\left(\mathrm{MA}^{+}\right.$cation) upon illumination. However, there have been no clear investigations for 
this hypothesis; thus, it remains as an under-researched subject. A year later, D. Almond et al. have provided a clarification for this kind of giant dielectric behavior in a porous PZT material drenched with water [76]. The study demonstrated that the real part of relative permittivity values were remarkably greater than $10^{8}$ at lower frequencies. In which, the occupied water became a conductive network with in the insulating pores-PZT host that increased the conductivity or dielectric loss of the sample. Similarly, in case of HP polycrystalline thin films, the perovskite crystallites are parted from one another by grain boundaries (GBs) or voids. The defects near GBs are the sources for trapping carriers, band bending, and depletion layers that result in GB capacitance. Thus, under illumination the perovskite crystallites surrounded by GBs and voids become electrically conductive due to rapid generation of charge carriers, which will amplify the dielectric loss factor there by the dielectric permittivity values. Therefore, the authors suggest that the light illumination on halide perovskite produces electrically conductive microstructures within the dielectric host, which is similar to that of water occupied porous PZT samples. Similarly, in our earlier study we have confirmed the light-effect on dielectric properties of $\mathrm{MAPbI}_{3}-\mathrm{PVDF}$ composite films [51]. As presented in Figure 2b,c, the dielectric properties of $25 \mathrm{vol} \% \mathrm{MAPbI}_{3}-\mathrm{PVDF}$ composite films were thoroughly investigated under dark and illuminations with various intensities of illumination $\left(0.06-3.23 \mathrm{~mW} / \mathrm{cm}^{2}\right)$. Where, the $\varepsilon_{\mathrm{r}}$ and $\mathrm{D}$ values were enormously increased with increasing intensity of light intensity compared to dark-state measurement. In which, under dark those values were approximately similar all over the frequency range, while abrupt changes were observed for composite films under illumination particularly in the lower frequency range $(0.1-1 \mathrm{kHz})$. This kind of giant dielectric behavior at low frequencies in $\mathrm{MAPbI}_{3}-\mathrm{PVDF}$ composite is because of rapid generation of photo-generated charge carriers in light-active $\mathrm{MAPbI}_{3}$ perovskite under illumination, which may adversely increase the conductivity of the sample leading to sudden increase in dielectric loss value. Besides, the light-effect on leakage current density properties of the same composite film was also investigated as depicted in Figure $2 \mathrm{~d}$. Where, the leakage current density of the composite film was gradually increased as the light intensity increases and reached to $\sim 10^{-4} \mathrm{~A} / \mathrm{cm}^{2}$ at an applied field of $100 \mathrm{kV} / \mathrm{cm}$ for used light intensity of $3.23 \mathrm{~mW} / \mathrm{cm}^{2}$, which is due to photo-generated charge carriers as aforesaid. The generated free charges not only make the conduction path but also become as the interfacial defects at the interfaces of sample and electrodes. This type of light-dependent dielectric behavior could be the reason for attained piezoelectric and triboelectric output performance changes in the HP-based nanogenerators upon illumination. Ferroelectric materials are a subclass of pyroelectric and piezoelectric materials and exhibit the switchable spontaneous polarization based on an applied electric field. Kutes et al. experimentally provided evidence for the ferroelectric nature of solution-processed HP films $\left(\beta-\mathrm{MAPbI}_{3}\right)$ for the first time while performing the piezo force microscopy (PFM) study [69]. There have not been many reports on the ferroelectric properties of HPs; however, numerous studies have proven the dielectric and piezoelectric properties of HP materials [65,70,71,77-79]. Particularly, commonly employed $\mathrm{MAPbI}_{3}$ perovskite shows an average piezoelectric coefficient $\left(\mathrm{d}_{33}\right)$ of $\sim 2.7 \mathrm{pm} / \mathrm{V}$ for a single crystal and within the range of 4-5.2 pm/V for polycrystalline films [70,71,77]. Subsequently, compositional tuning of HPs by a simple solution process has offered a promising platform to design various HPs with enhanced dielectric, ferroelectric, and piezoelectric properties $[65,78,79]$. For example, the synthesized $\mathrm{FAPbBr}_{3}$ nanoparticles fabricated using the solution method demonstrated notable improvement in ferroelectric properties with high $\mathrm{d}_{33}$ of $\sim 25 \mathrm{pm} / \mathrm{V}$ [78], while inorganic $\mathrm{CsPbBr}_{3}$ film also revealed a higher $\mathrm{d}_{33}$ value $(7.7 \mathrm{pm} / \mathrm{V})$ and improved stability than $\mathrm{MAPbI}_{3}$ films [79], whereas the HP-polymer composite materials reveal significantly larger piezoelectric properties than neat HP materials due to improved dielectric properties caused by MWS or interfacial polarizations as above discussed [44,45,51]. Moreover, the composites possess good mechanical properties and flexible characteristics which benefit them for continuous operation in harsh conditions. With these advantageous features, $\mathrm{HP}$-polymer composite based nanogenerators are exhibiting remarkable output perfor- 
mances that are comparable to those inorganic materials based nanogenerators [44,51,79]. Likewise, the contact electrification effects, generated due to interaction with other triboelectric materials, have been thoroughly investigated for the HP films after the first report on $\mathrm{MAPbI}_{3}$ films [80]. Additionally, the triboelectric properties of HP materials can be remarkably altered via compositional tuning. For example, Wang et al. demonstrated that the surface potential of $\mathrm{CsPbBr}_{3}$ perovskite material changes owing to significant $\mathrm{Ba}^{2+}$ doping in controlled quantity [81]. Therefore, HPs have proven to be highly significant in various nanogenerators, including PENGs, TENGs, and PyENGs, to harvest several unused energy sources such as mechanical vibrations and thermal energies due to their excellent dielectric properties $[65,67,68]$. These nanogenerators, influenced by the applied energy, provide source-dependent outputs. Thus, they are considered as self-powered sensors to identify the applied degree of the source. However, coupling dielectric, piezoelectric, pyroelectric, or triboelectric characteristics of HPs with their extraordinary photoactive nature demonstrate their great potential to develop efficient multifunctional devices for harvesting energies and to detect external signals in self-powered mode. This review emphasizes and summarizes HP-based nanogenerators and their role as self-powered sensors to recognize various external stimuli, such as temperature, pressure, light, and some of the physiological signals (Figure 1).

a

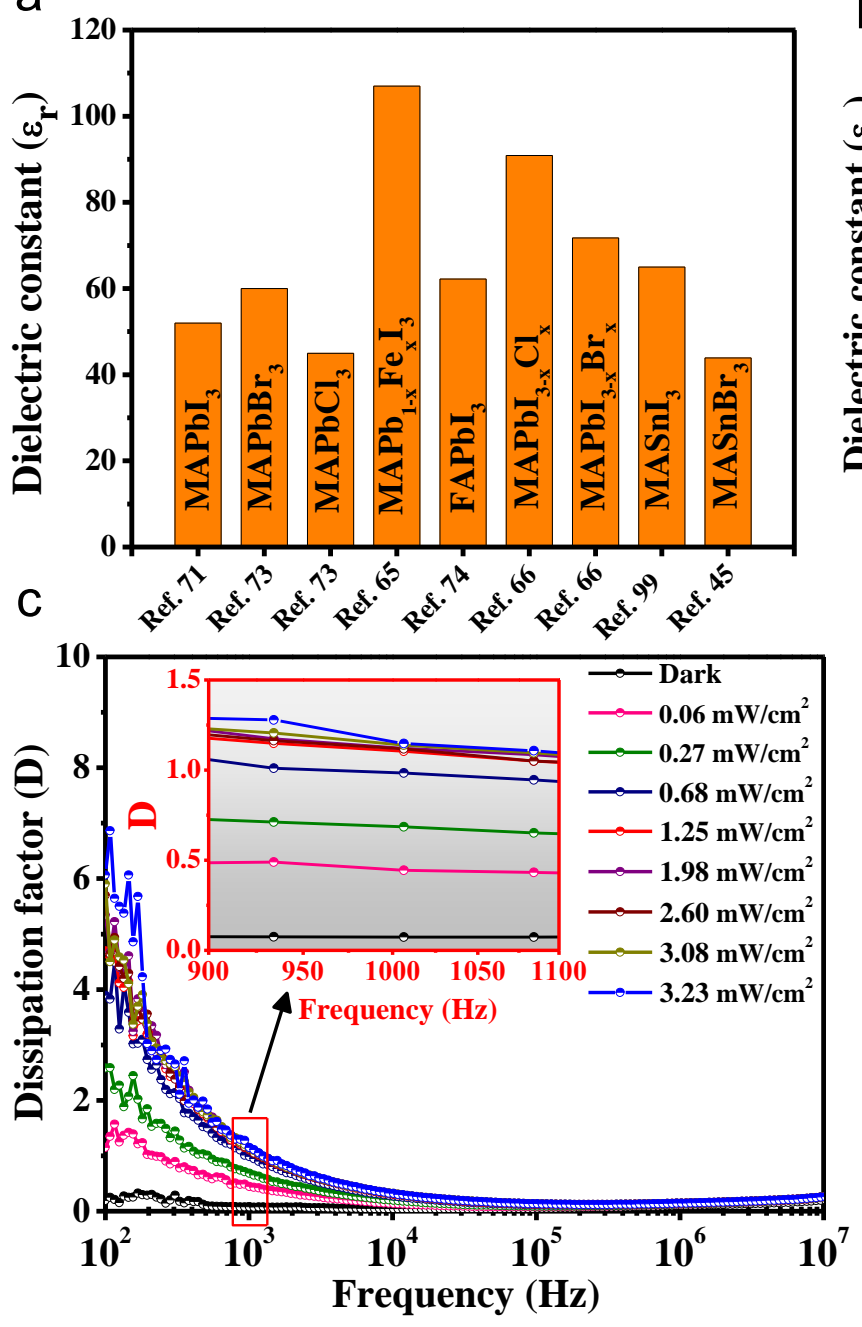

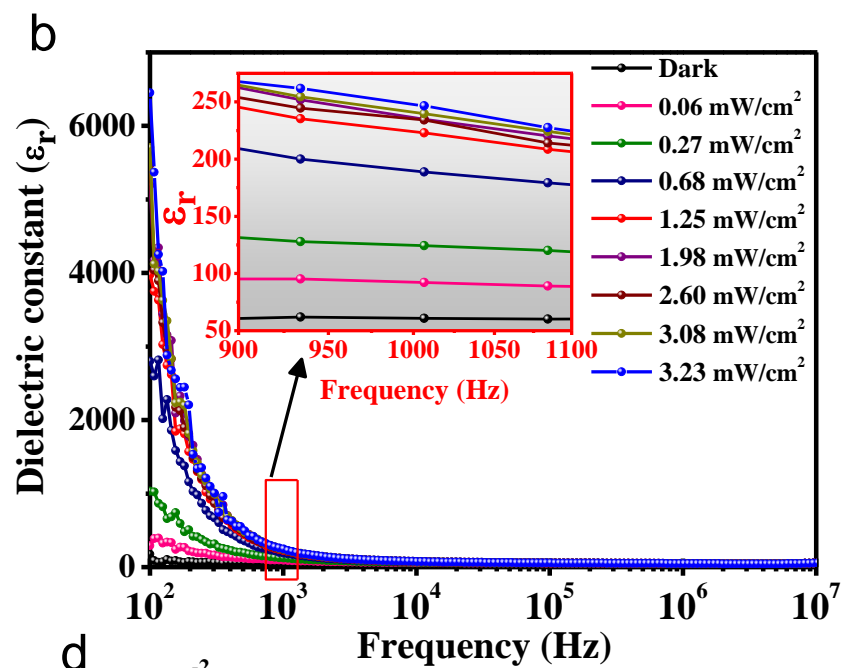

d

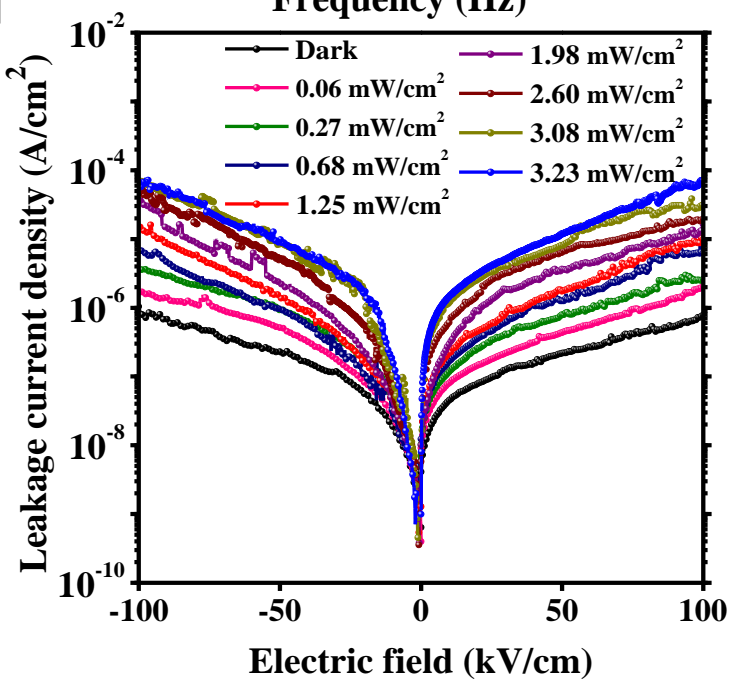

Figure 2. (a) Dielectric constants of various frequently used HP materials. Light intensity effect on frequency dependent (b) dielectric constant and (c) dissipation factor of $\mathrm{MAPbI}_{3}-\mathrm{PVDF}$ composite films. (d) Light intensity effect on leakage current density of $\mathrm{MAPbI}_{3}-\mathrm{PVDF}$ composite films as a function of applied electric field. (b-d) Reprinted with permission from ref. [51], Copyright 2020, American Chemical Society. 


\subsection{Operational Mechanism of HP-Based Nanogenerators as Self-Powered Sensors}

Nanogenerators that produce electricity from renewable sources are distinctly categorized as PENG, TENG, and PyENG based on their working mechanisms. They distinctly operate as self-powered sensors, including pressure/strain and temperature sensors. These nanogenerators produce a measurable quantity of electrical signals in response to external mechanical energy created by factors such as vibrations, biomechanical moments, acoustic waves, and wind, and waste heat or thermal energy from the surroundings. HP materials have received great attention to fabricate self-powered pressure/strain sensors, temperature sensors, and photodetectors owing to their impressive piezoelectric, pyroelectric, and optoelectronic properties. The working mechanisms of nanogenerators to sense pressure/strain, temperature, and light are briefly discussed in the following section.

\subsubsection{Mechanism of HP-based PENG as a Self-Powered Sensor}

PENG-based self-powered pressure/strain sensors can readily detect and quantify the applied mechanical vibrations with a broad range of applications in various fields, such as health monitoring, electronic skin, wind speed detection, and human-machine interface [82-85]. PENG converts applied mechanical energy into electrical energy based on the piezoelectric effect, typically referred to as a generation of electric potential under applied pressure or vice versa. The working mechanism of HP-based PENG as a selfpowered pressure and a photodetector is deliberately explained based on the previous literature, as shown in Figure 3a,b, respectively [51]. When a mechanical force is applied to the PENG, the deformation of piezoelectric material generates polarization by forming dipoles in the active layer. Therefore, a piezoelectric potential difference is created between both the electrodes, pushing the electrons from one electrode to another via the external circuit producing a positive signal (Figure 3a). Subsequently, generated piezoelectric potentials would vanish when the mechanical force is released from the PENG. Therefore, the unbalanced potential difference allows electrons to flow back to the initial state through the external circuit; thus, producing a negative signal. However, the piezoelectric output from PENG is greatly influenced by operating conditions, such as applied pressure and frequency. Particularly, the pressure-dependent output characteristics of PENG facilitate the device's operation as a pressure or strain sensor. Similarly, the same HP-based PENG can be operated as a photodetector owing to its continuous, and significant variations in output upon illumination. When the HP-based PENG is illuminated during its operation, the deformation of HP under strain generates dipoles, while the illumination produces electron-hole pairs owing to the light-active nature of HPs (Figure 3b). This photogenerated charge carries increases the leakage current/photoconductivity of the perovskite. Therefore, the generated piezoelectric potentials across the device decrease, leading to diminished piezoelectric output. The light-stimulated piezoelectric outputs of HP-based PENG under controllable illuminations and constant applied pressure enable it as a selfpowered photodetector. However, extracting light- and pressure-dependent electric signals from HP-based PENG without signal interference makes it feasible to operate PENG as a bimodal sensor for simultaneous detection of pressure and lights [51].

\subsubsection{Mechanism of HP-Based TENG as a Self-Powered Sensor}

TENG can effectively transform the irregular and randomly distributed mechanical energy into usable electricity via coupling contact electrification with electrostatic induction [87]. TENG-based pressure/strain sensors have the potential to be used to sense movement monitoring, vibration, pressure, and touch [21,88-90]. Figure $3 c, d$ shows the working mechanism of HP-based TENG as a self-powered pressure sensor and a photodetector, respectively based on the vertical contact-separation mode- the first fundamental TENG operational mode [86]. When the mechanical force is applied on the TENG, the two dissimilar triboelectric materials-one is perovskite and the other is different materialhave contact electrification at the interface, generating an equal quantity of triboelectric charges with opposite polarities on both the surfaces due to difference in their electron 
affinities. For example, in case of HP-carbon TENG, positive and negative charges appear on the surfaces of HP and carbon materials, respectively upon contacting each other due to difference in their electron affinities (Figure $3 c, d$ ). These surface triboelectric charges induce charges onto the electrodes with opposite polarity via the electrostatic induction effect when both the surfaces start to separate from each other, building electric potential difference between the electrodes. Subsequently, electrons flow through the external circuit to balance the potential difference, producing an electrical output signal (Figure 3c). When both the surfaces are fully separated, electric potentials reach an equilibrium state, restricting the further movement of electrons through the circuit. However, when the top surface approaches the surface of HP again, the unbalanced electric potentials in both the electrodes drive the electrons to flow back via an external circuit; thus, generating the electrical signal with opposite polarity. If the TENG is illuminated during its operation, numerous electron-hole pairs are generated in light-active HP (Figure 3d). On the other hand, the contact electrification between HP and counter triboelectric material creates the triboelectric charges on both surfaces with opposite polarities. The generated photo charge carriers significantly influence the surface charge density of the triboelectric materials based on the type of materials used as counterparts in TENG $[52,80,81,86]$. For instance, triboelectric positive charges are generated on perovskite surface in FTO/perovskite//carbon/Ag TENG, while negative charges appear on carbon surface upon contacting owing to different electron affinities. On the other hand, a depletion region having a built-in electric field with an upward direction (from HP to carbon) is generated at their interface when the carbon surface comes in full contact with the perovskite surface. Furthermore, electron-hole pairs are generated once the device is illuminated. The formed built-in electric field prompts the separation of photogenerated electron-hole pairs pushing the flow of electrons and holes towards the bottom electrode (FTO) and towards the surface of the perovskite, respectively. Therefore, the combined photo charge carriers with triboelectric charges increase the surface charge density on the perovskite surface, leading to significant enhancement in TENG output performance under the illumination than the dark-state operation. Additionally, a similar trend in triboelectric output performance is observed in TENG with $\mathrm{FTO} / \mathrm{TiO}_{2} /$ perovskite//PTFE/ Cu/Sub [52]. The photogenerated electrons move toward the electron transport $\mathrm{TiO}_{2}$ layer leaving the holes at the surface of perovskite, resulting in improved triboelectric output performance. On the contrary, they observed the decreased outputs for the same device under illumination when a triboelectric positive aluminum was used instead of PTFE-triboelectric negative material. When both the surfaces contact each other, triboelectric positive and negative charges appear on the surfaces of $\mathrm{Al}$ and perovskite, respectively, because of the strong triboelectric positive nature of Al. The photogenerated holes neutralize the triboelectric negative charge on the surface of perovskite under illumination and adversely affect the output. HP-based TENGs can be used as selfpowered sensors owing to their pressure and light-controlled outputs (either single-mode or bimodal sensor) to detect pressure and light signals simultaneously or individually like HP-based PENGs. However, more theoretical and experimental studies are required to understand the working mechanism of HP-based TENGs under illumination in detail.

\subsubsection{Mechanism of HP-Based PyENG as a Self-Powered Sensor}

PyENG is an energy harvesting device that harvests waste heat and converts it into electrical energy via the pyroelectric effect $[23,91]$. The pyroelectric effect is defined as the change in spontaneous polarization of certain material in response to the temperature change. The working mechanism of PyENG is discussed according to the literature as follows [92,93]. No polarization occurs in the pyroelectric material in the absence of temporal change in temperature in the device $(\mathrm{dT} / \mathrm{dt}=0)$. Therefore, it prevents the generation of pyroelectric output. However, the total spontaneous polarization decreases with the increasing temperature, resulting in the electron flow from top to bottom electrodes through the external circuit. Therefore, it generates an electric output signal. Once the temperature reaches the equilibrium state, no pyroelectric output is generated due to the 
saturated potential difference between electrodes. However, the spontaneous polarization of the crystals increases when the device is cooled down due to temperature fluctuations, leading to a change in potential difference. Hence, electrons move back through the external circuit from the bottom to the top electrode by generating the opposite output signal until it reaches the equilibrium state. This generation of the pyroelectric output signal is a cyclic process under applied cyclic temperature changes. Therefore, PyENG can be used as a temperature sensor owing to the output signal generated based on temperature change to distinguish the temperature changes. However, there have been no studies reported so far on the effect of light on pyroelectric properties of HP materials or their PyENGs.

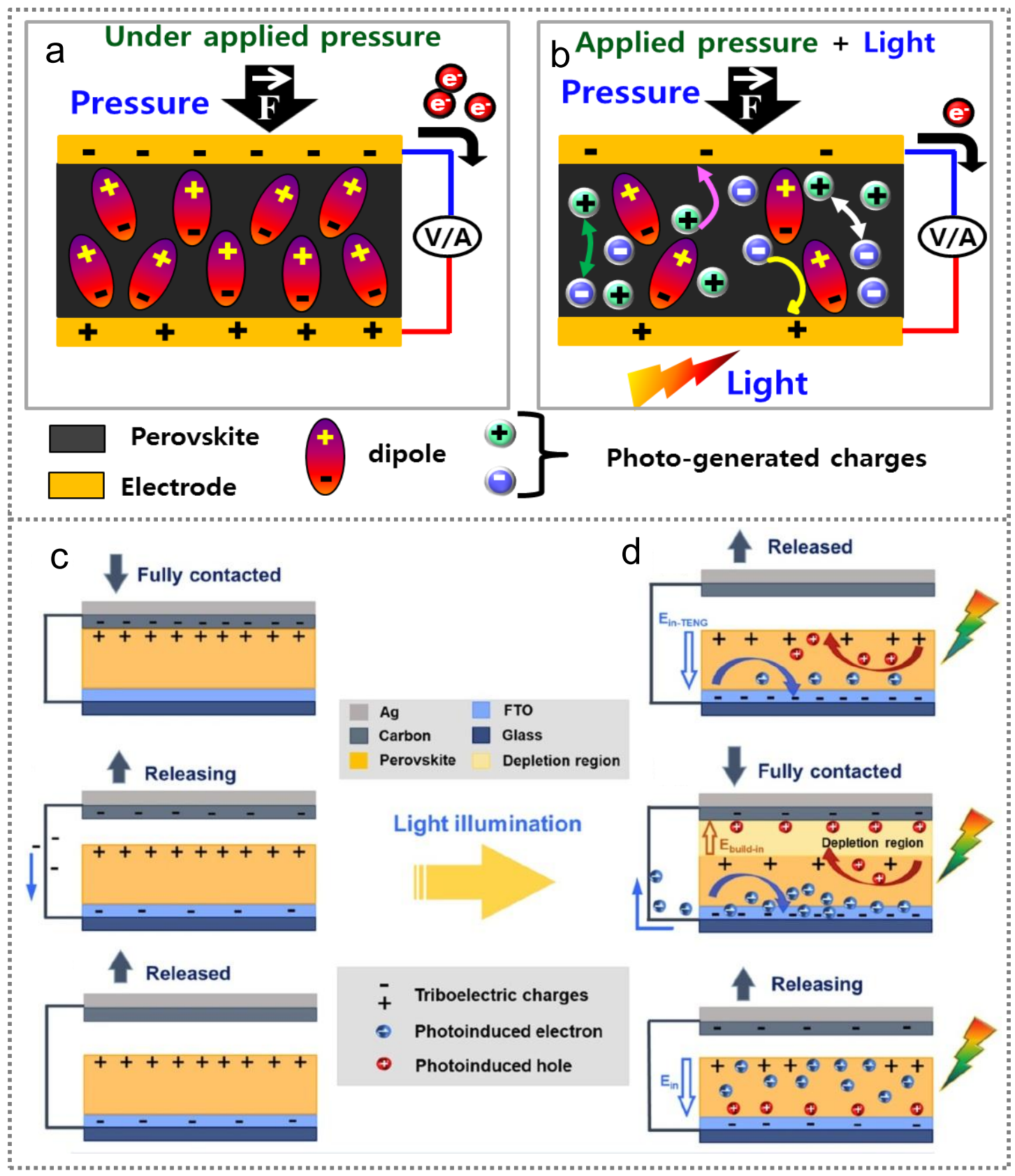

Figure 3. Operational mechanism of HP-based PENG as a self-powered sensor: (a) pressure sensor and (b) photodetector. Operational mechanism of HP-based TENG as a self-powered sensor: (c) pressure sensor and (d) photodetector. (c,d) Reprinted with permission from ref. [86], Copyright 2020, Elsevier. 


\subsection{Halide Perovskite Nanogenerator Based Self-Powered Sensors}

\subsubsection{Temperature Sensors}

Temperature sensors are needed in various fields, such as environment monitoring, thermal imaging, biomedical sensing, and thermal monitoring of microprocessors [94,95]. According to the previous studies, HP materials generally reveal temperature-dependent structural/phase transitions, which can be reflected in their photoluminescence spectra [96-98]. This phenomenon extends their applicability in the field of temperature sensor technology. However, these devices require steady spatial temperature variations. Moreover, the performance of such systems is significantly affected when the device encounters temperature variations with time rather than with spatial distribution. Nevertheless, PyENGs convert time-varying temperature fluctuations into usable electricity. Sultana et al. reported the first pyroelectric nanogenerator (PyENG) based on HP-polymer composite material and observed the temperature-dependent output performance [48]. Their nanogenerator was comprised of $\mathrm{MAPbI}_{3}-\mathrm{PVDF}$ nanofibers mat that was prepared by electrospinning of $\mathrm{MAPbI}_{3}-\mathrm{PVDF}$ composite precursor solution at an applied tip voltage of $6.7 \mathrm{kV}$. The electroactive $\beta$-phase of PVDF polymer significantly augmented up to $\sim 95 \%$ when the $\mathrm{MAPbI}_{3}$ was infiltrated into the PVDF matrix owing to the possible interactions between HP filler material and PVDF polymer. The neat PVDF films exhibited only up to $\sim 70 \%$ of the $\beta$-phase. According to the authors, the plausible interactions happened between the iodine atoms from the $\mathrm{PbI}_{3}-$ framework of perovskite and the $-\mathrm{CH}_{2}$ dipoles of PVDF. The improved electroactive $\beta$-PVDF phase would amend the piezoelectric and pyroelectric output performances of nanogenerators. The pyroelectric nanogenerator (PyENG) was fabricated by attaching the $\mathrm{Ni}$ and $\mathrm{Cu}$-coated fabrics as top and bottom electrodes onto the $\mathrm{MAPbI}_{3}-\mathrm{PVDF}$ nanofibers mat, respectively, followed by wiring with copper wires (Figure 4a). Finally, the generator was sealed with a polypropylene sheet to increase the mechanical endurance of the device. The pyroelectric performance of the nanogenerator was studied by measuring the output voltage and currents under applied temperature changes across the generator with cyclic heating and cooling system. A positive output current signal was detected with a value of $\sim 15.7 \mathrm{pA}$ after the temperature was raised from room temperature to $336 \mathrm{~K}$. However, a negative signal with $\sim 18.2 \mathrm{pA}$ was obtained after the system cooled down to RT (Figure $4 b$ ). The pyroelectric coefficient (p) of the $\mathrm{MAPbI}_{3}-\mathrm{PVDF}$ composite was calculated to be $\sim 44 \mathrm{pC} / \mathrm{m}^{2} \mathrm{~K}$ from the output current. The nanogenerator demonstrated a fast response time of $\sim 1.14 \mathrm{~s}$ and good reversibility for output changes throughout the cyclic heating and cooling. Additionally, the linear relationship trend between pyroelectric output performance and $\mathrm{dT} / \mathrm{dt}$ of the device suggests its potential use as a self-powered temperature sensor in detecting the temperature changes (Figure 4c). However, unlike thermoelectric generators (TEGs) these HP-based PyENGs cannot be applicable to sense the temperature when there is no change in temperature or constant temperature $(\mathrm{dT} / \mathrm{dt}=0)$ since no polarization occurs in the pyroelectric material. Moreover, HP materials have low Curie temperature (Tc) compared with the conventional ceramic perovskites. For instance, commonly used tetragonal $\mathrm{MAPbI}_{3}$ perovskite exhibited $\mathrm{T}_{\mathrm{c}}$ of $330 \mathrm{~K}$ [71]. Further, the $\mathrm{T}_{\mathrm{c}}$ of $\mathrm{MAPbI}_{3}$ films decreased to $320 \mathrm{~K}$ with partial incorporation of $\mathrm{Fe}$ into $\mathrm{Pb}$ sites [65]. Although these materials showed the impressive pyroelectric properties, relatively low $\mathrm{T}_{\mathrm{c}}$ (i.e., just above the room temperature) of these HP materials limited their application in pyroelectric devices at higher temperature. In addition, stability issue of HPs is another major reason that impeding the practical application of HP-based pyroelectric devices. However, there have not been many studies elucidating the pyroelectric properties of these HP materials. The research in the field of pyroelectric nanogenerators or temperature sensors based on the pyroelectric effect of HPs is still under consideration. 


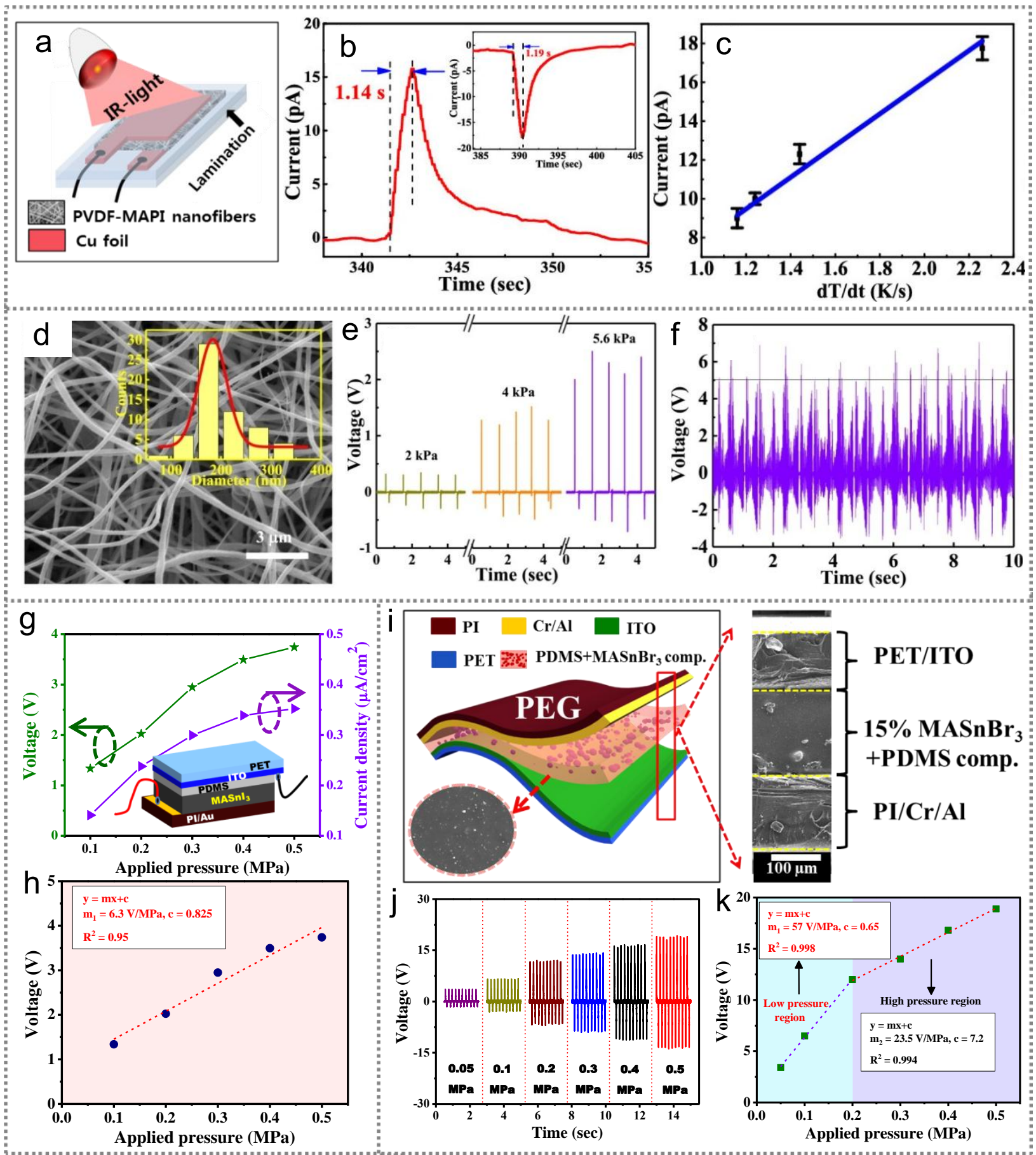

Figure 4. (a) Schematic illustration of $\mathrm{MAPbI}_{3}-\mathrm{PVDF}$ composite-based PyENG, (b) corresponding pyroelectric output signal measured at varied temperature range from 298 to $336 \mathrm{~K}$, and (c) relationship between obtained pyroelectric outputs with dT/dt. (a-c) Reprinted with permission from ref. [48], Copyright 2019, American Chemical Society. (d) Surface morphology of electrospun $\mathrm{MAPbBr}_{3}-\mathrm{PVDF}$ composite nanofibers, (e) pressure dependent output voltages of acoustic nanogenerator, and (f) output voltage from the nanogenerator under an applied sound pressure of $85 \mathrm{~dB}$. (d-f) Reprinted with permission from ref. [50], Copyright 2018, Elsevier. (g) Pressure dependent peak output voltage and current density values and (h) pressure sensitivity in terms of output voltage of $\mathrm{MASnI}_{3}$-based PENG. (g,h)Reprinted with permission from ref. [99], Copyright 2019, Elsevier. (i) Schematic diagram and SEM cross-section image of $\mathrm{MASnBr}_{3}-\mathrm{PDMS}_{\text {composite-based }}$ PENG. Inset shows the surface morphology of the composite film. (j) Corresponding pressure dependent piezoelectric output signals and (k) pressure sensitivity in terms of output voltage. (i-k) Reprinted with permission from ref. [45], Copyright 2020, American Chemical Society. 


\subsubsection{Pressure Sensors}

A pressure sensor is one of the essential devices for wearable technology; it has a wide range of applications, such as motion detection, human-machine interaction, speakers, robotics, and e-skin devices [100-103]. Flexible pressure sensors based on the piezoelectric effect have gained considerable attention owing to the facile fabrication methods, low cost, and ease of acquiring electrical signals. The nanogenerators based on piezoelectric materials can be considered as both power sources and self-powered pressure sensors due to their pressure-dependent response [104-114]. Although there are several piezoelectric materials to obtain piezoelectric pressure sensors, recently progressed HP materials have gained considerable attention from researchers not only due to their dielectric and piezoelectric properties but also their facile and low-temperature synthesis routes than other ceramic materials $[44,115,116]$. Additionally, the piezoelectric acoustic nanogenerator was reported based on flexible and lightweight $\mathrm{MAPbBr}_{3}-\mathrm{PVDF}$ composite nanofibers mat that fabricated via electrospinning procedure has the majority of electroactive $\beta$-PVDF phase and good crystallinity [50]. The morphology shown in the SEM surface image of Figure $4 \mathrm{~d}$ reveals the relatively decent quality of nanofibers without any beads or cracks. Additionally, incorporating $\mathrm{MAPbBr}_{3}$ into the PVDF matrix significantly enhances the electroactive $\beta$-phase and crystallinity of PVDF polymer due to the beneficial interactions between perovskite and polymer, as mentioned above. Importantly, during in situ electrospinning process or poling process, the induced charge onto the perovskite attracts PVDF molecules and facilitates their orientation in $\beta$-crystalline form by acting as a nucleating agent. The fabricated $\mathrm{MAPbBr}_{3}$-PVDF composite-based PENG exhibited an output voltage and currents of $\sim 5 \mathrm{~V}$ and $60 \mathrm{nA}$, respectively, under finger touch with an applied pressure of $9.8 \mathrm{kPa}$. Moreover, the outputs of PENG increased linearly with an applied strain in the range of 2 to $5.6 \mathrm{kPa}$, as shown in Figure 4e. It showed the pressure sensitivity of $0.1 \mathrm{~V} / \mathrm{Pa}$, making it more appropriate to identify the low-level pressures or finger touch. Additionally, the device response was verified at a sound pressure level of $85 \mathrm{~dB}$, obtaining the output voltage of $\sim 5 \mathrm{~V}$, to comprehend the feasibility of the nanogenerator as an acoustic sensor (Figure 4f). The acoustic nanogenerator demonstrated a significantly higher acoustic sensitivity of $\sim 13.8 \mathrm{~V} / \mathrm{Pa}$, making it a high potential candidate for a self-powered acoustic sensor to detect the surrounding noise pollution levels. Further, many reports have addressed the viability of HP/HP-polymer composite-based nanogenerators as selfpowered pressure sensors $[45,65,99,117-120]$. For example, the lead-free MASnI $_{3}$-based flexible PENG generated an output voltage of $3.8 \mathrm{~V}$ at an applied pressure of $0.5 \mathrm{MPa}$ (Figure 4g) [99]. Additionally, the output of the same device was gradually increased by increasing the applied pressure from 0.1 to $0.5 \mathrm{MPa}$. It exhibited an approximately linear relationship between applied pressure and output voltage with a pressure sensitivity value of $6.3 \mathrm{~V} / \mathrm{MPa}$ as shown in Figure $4 \mathrm{~h}$. As illustrated in Figure 4i, the flexible and eco-friendly nanogenerator based on predominant air-stable $\mathrm{MASnBr}_{3}$-PDMS composite is quite capable of achieving a higher piezoelectric output performance of $\sim 18.8 \mathrm{~V}$ than a neat $\mathrm{MASnBr}_{3}$ device [45]. An inset SEM image in Figure 4i shows the uniform dispersion of $\mathrm{MASnBr}_{3}$ particles in the PDMS matrix. However, the increased harvesting capability of the $\mathrm{MASnBr}_{3}-\mathrm{PDMS}$ nanogenerator is attributed to the improved dielectric properties owing to the larger interfacial polarization. Additionally, its output voltage was gradually increased with increasing the applied pressure from 0.05 to $0.5 \mathrm{MPa}$ (Figure 4j). The device response to the applied pressure was different for different level of input pressures. For example, the device demonstrated a superior response with a sensitivity value of $57 \mathrm{~V} / \mathrm{MPa}$ in the low-pressure region $(0.05$ to $0.2 \mathrm{MPa})$ than in the high-pressure region $(23.5 \mathrm{~V} / \mathrm{MPa})$ as shown in Figure $4 \mathrm{k}$. Furthermore, the recently reported double halide perovskites (e.g., $\mathrm{TMCM}_{2} \mathrm{SnCl}_{6}$ ) have also demonstrated the pressure-sensitive output performance [120]. Such a pressure-sensitive output variation of PENGs under the change in applied pressure makes them a potential candidate for self-powered flexible pressure or tactile sensor with wider utility in wearable electronics. The essential parameters including sensitivity values of the HP-nanogenerator based pressure sensors are summarized in 
Table 1. However, HP-based pressure sensors reported until today are mainly operated under very low-level pressure conditions $(\leq 500 \mathrm{kPa})$, which may be a limitation for these sensors to be operated in high-level pressure regions or harsh environments. In addition, most of the HP-based nanogenerators revealed a high sensitivity at extremely low-pressure range but they exhibited nearly saturated outputs at applied high pressures ( $>300 \mathrm{kPa})$, which can be another drawback of these sensors as they can give nearly lower pressure sensitivity values in high pressure regions as afore discussed. However, HP-polymer based materials can be employed for constructing pressure sensors as they can be predominantly operated in harsh conditions due to their excellent mechanical and flexible characteristics.

\subsubsection{Physiological or Biomechanical Sensors}

Self-powered sensors have been actively employed as implantable biomechanical sensors to monitor the biological motions or biomedical signals, such as heart rate, blood pressure, and respiratory rate. The nanogenerators can be helpful to harvest the motion energy of organs and have the ability to sense biomechanical movements simultaneously. Several HP-based nanogenerators have been used as physiological or biomechanical sensors by immediately securing the device onto the human or animal body. For example, in our previous study, we employed halide $(\mathrm{Cl} / \mathrm{Br})$ incorporated $\mathrm{MAPbI}_{3}$ perovskite as an active piezoelectric material and reported the flexible PENG to harvest the energy from biomechanical motions, as shown in Figure 5a [66]. Among many, the nanogenerator based on $4 \mathrm{Cl}-\mathrm{MAPbI}_{3}$ perovskite exhibited higher piezoelectric output performance due to its enormous dielectric and piezoelectric properties. Furthermore, the device was successfully subjected to the human body to harvest various human motions, such as finger and wrist motions with $\sim 2.6 \mathrm{~V}$ and $\sim 0.07 \mathrm{~V}$, respectively (Figure $5 \mathrm{~b}, \mathrm{c}$ ). Such a motion-sensitive response benefits the device to be considered for a biomechanical or physiological sensor to sense human motions. An eco-friendly nanogenerator based on lead-free $\mathrm{MASnBr}_{3}-$ PDMS composite was also considered to be a potential candidate for harvesting several biomechanical motions, such as walking, finger, elbow, and wrist movements [45]. The authors suggested, based on the outcomes, that such motion-sensitive response of device make the device a potential candidate for a self-powered biomechanical sensor to be used as wearable health-monitoring devices. Furthermore, Kim et al. described the utility of inorganic $\mathrm{HP}$ material $\left(\mathrm{CsPbBr}_{3}\right)$ for electromechanical energy harvesting as well as for recognizing the body movements specifically [79]. The nanogenerator with $\mathrm{PET} / \mathrm{ITO} / \mathrm{PDMS} / \mathrm{CsPbBr}_{3} / \mathrm{ITO} / \mathrm{PET}$ structure was fabricated and was applied to the poling process with an applied electric field of $25 \mathrm{kV} / \mathrm{cm}$. The used $\mathrm{CsPbBr}_{3}$ film displayed the enormous piezoelectric properties $\left(\mathrm{d}_{33}=40.3 \mathrm{pm} / \mathrm{V}\right)$ after the optimized poling process owing to the octahedral $\left(\mathrm{PbBr}_{6}\right)$ distortions of a unit cell under poling field. As shown in Figure 5d, the device was further secured onto the human body and sensed selective regular physiological motions, such as eye-blinking, throat actions (coughing and yawning), and finger motions. The generator could produce distinguishable electrical current signals ( 0.6 nA) under eye-blinking motion depending on the frequency of blinking-one time or multiple times (Figure 5e). Similarly, the device was successful in identifying throat and finger motions by producing discrete electrical signals for each kind of movement (Figure 5f). The results demonstrate that the dissimilar electrical signals from the generator correspond to different motion of body parts, implying the potential physiological sensing capability with higher sensitivity of $\mathrm{CsPbBr}_{3}$-based nanogenerators in a self-powered way to identify various body motions. Additionally, other research groups demonstrated a flexible, self-powered neural-stimulating e-skin based on photosensitivetriboelectric $\mathrm{MAPbI}_{3}-\mathrm{PDMS}$ composite films (Figure 5g) [121]. The e-skin operates based on the triboelectric effect and is effective in harvesting mechanical energy (i.e., human body actions) and in generating neural-stimulating electrical signals without the need for any external power supply. Under bending deformation, the prepared e-skin displayed a gradual increment in triboelectric output performance with increasing bending angle and obtained higher output for the bending angle of $60^{\circ}$ with the voltage and currents of 
$0.659 \mathrm{~V}$ and $8.94 \mathrm{nA}$, respectively. The device can be considered as a self-powered pressure sensor owing to such as strain-dependent behavior. Moreover, the device exhibited lightstimulated changes in triboelectric output performance owing to the photoelectric nature of $\mathrm{MAPbI}_{3}$ perovskite, as shown in Figure 5h. The device could be operated as a physiological sensor in a self-powered way owing to such light-stimulated energy harvesting characteristics. For example, the demonstrated animal experiment study represents the operation of prepared e-skin as a physiological sensor for in vivo characterization of synaptic plasticity, as illustrated in Figure 5i. Authors connected the e-skin to the hippocampus of the mouse brain to demonstrate the sensing application mentioned before. The e-skin was driven by several trivial body movements, and the output was controlled by light simultaneously recording the field excitatory postsynaptic potentials (fEPSP) (Figure 5j). The electrical neural-stimulating process on the mouse brain was controlled by the light on/off process; the process functioned as a wireless switch to modulate the neural stimulations. This study suggests that the $\mathrm{MAPbI}_{3}-\mathrm{PDMS}$ e-skin is successful in producing the postsynaptic responses for in vivo characterization of synaptic plasticity. Prominently, the e-skin attached to the human body can be used as a power source by scavenging the human motions, producing specific neural-stimulating electrical signals for the human brain without relying on a battery (Figure 5k). Such light-operational e-skins are helpful to fabricate self-powered wireless-controlled physiological sensor units for biomedical and neuroscience applications. The essential parameters of the HP-nanogenerator based physiological sensors are summarized in Table 1. All these reports clearly indicate that these HP materials are highly useful in identifying the biomechanical or physiological signals efficiently.

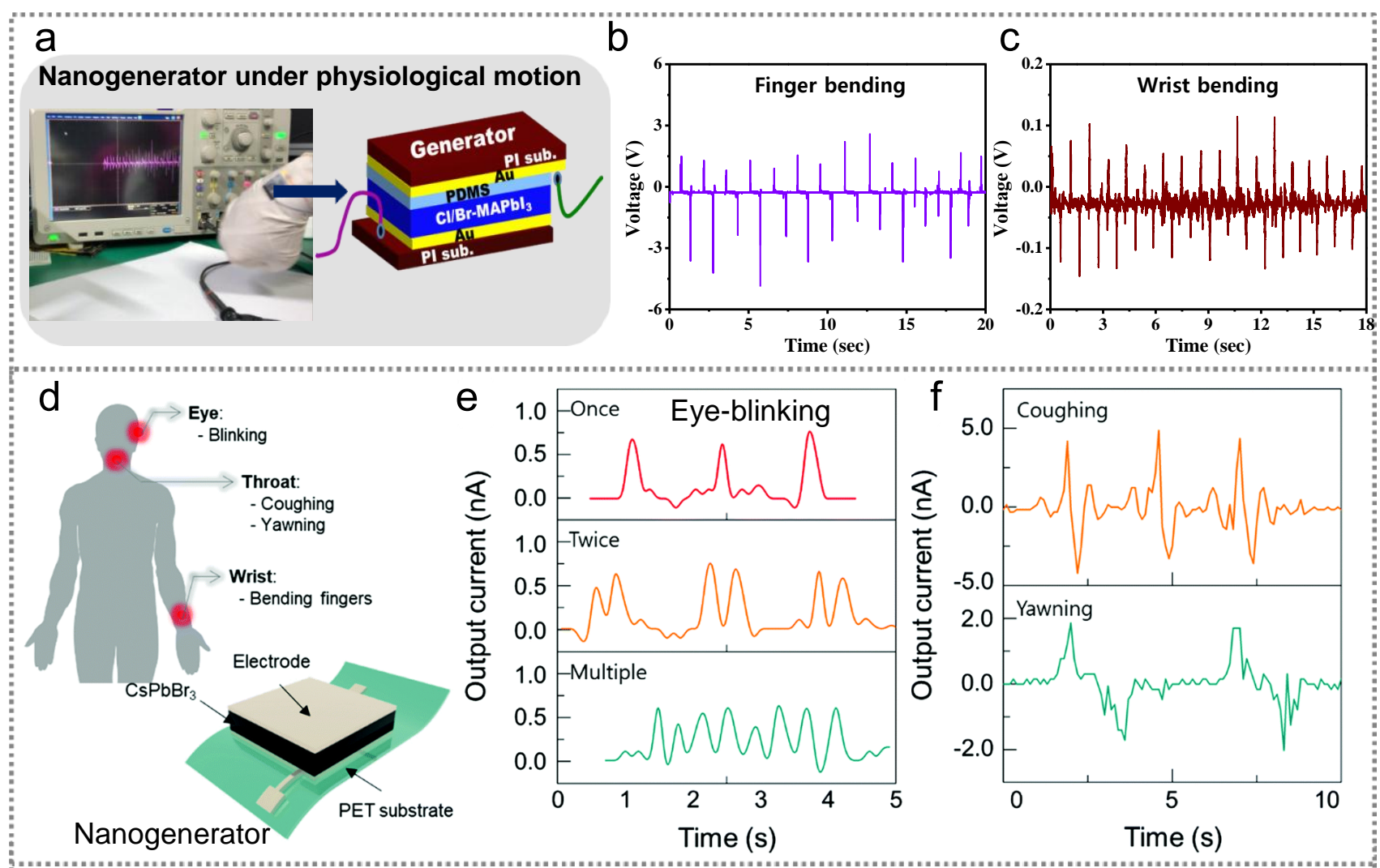

Figure 5. Cont. 


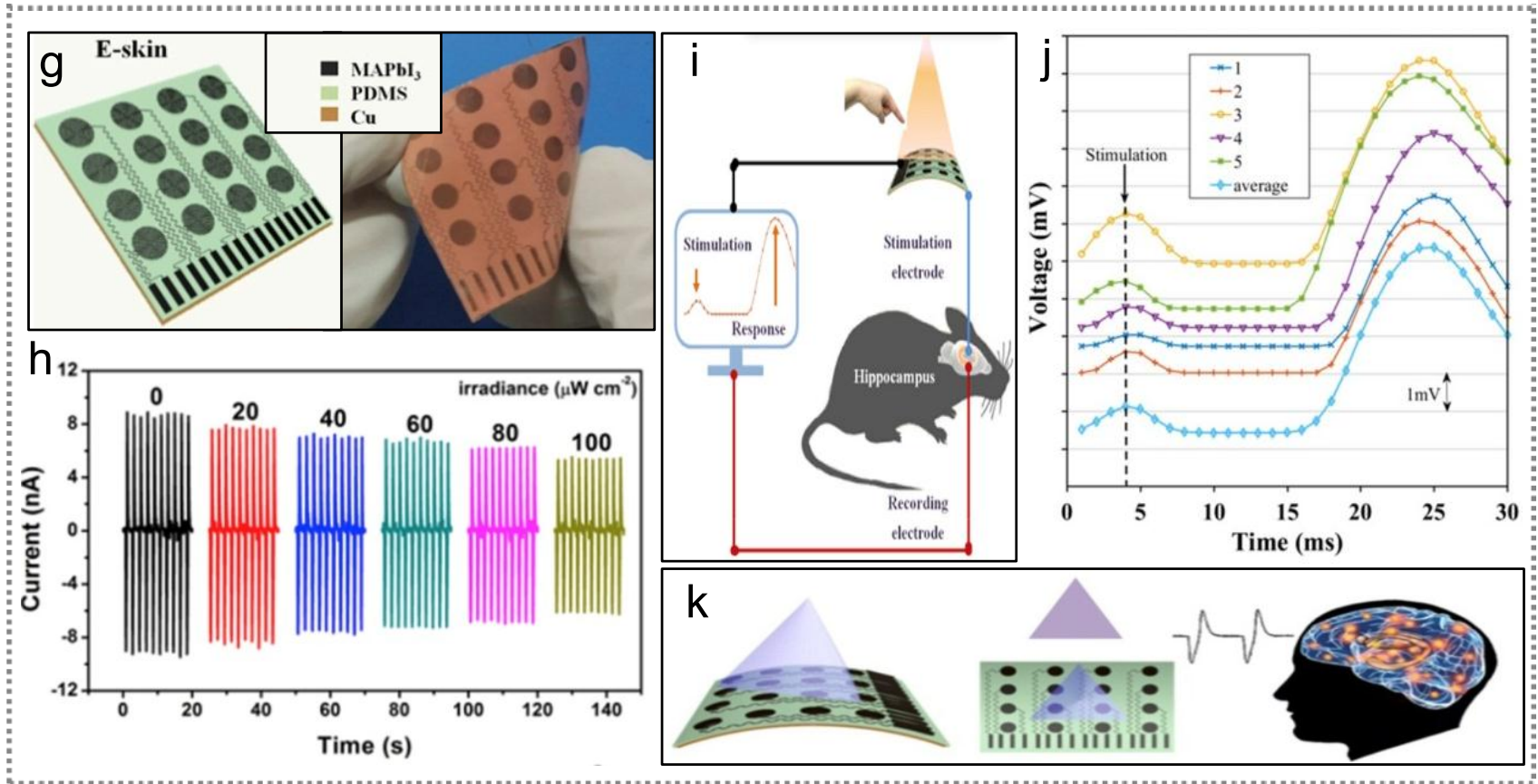

Figure 5. (a) Schematic illustration of halide-doped $\mathrm{MAPbI}_{3} \mathrm{PENG}$ with photographs while it is attached on the finger and wrist and $(\mathbf{b}, \mathbf{c})$ corresponding output voltage signals generated due to finger and wrist motions. (a-c) Reprinted with permission from ref. [66], Copyright 2020, American Chemical Society. (d) Schematic diagram of CsPbBr 3 -based PENG attached to the human body to monitor physiological movements, (e,f) corresponding electrical signals generated due to eye blinking and throat motions, respectively. (d-f) Reprinted with permission from ref. [79], Copyright 2020, The Royal Society of Chemistry. (g) Schematic and fabricated images of $\mathrm{MAPbI}_{3}$-based e-skin, (h) corresponding light-controlled triboelectric output under various light intensities, (i) the schematic sketch of animal experiment study using e-skin linking to mouse brain for synaptic analysis, (j) corresponding recorded field excitatory postsynaptic potentials (fEPSP) signals, and (k) schematic diagram of the e-skin as a photo wireless control unit for neural simulation. (g-k) Reprinted with permission from ref. [121], Copyright 2020, Elsevier.

\subsubsection{Photodetectors}

Photodetector (PD) is another most significant and widely accessed sensor in diverse fields, such as digital imaging, sensor networks, and optical communications [122]. HP materials with enormous optoelectronic properties have emerged as potential entrants in developing efficient PDs and self-powered PDs [123,124]. There have been many reports on HP-based self-powered photodetectors (SPDs) with different mechanisms/structures [125-130]. However, the SPDs based on nanogenerators have gathered remarkable attention owing to the enormous device stability, flexible characteristics, and easy and cost-effective fabrication methods, unlike the solar cell structured PDs [51-54]. Both PENG and TENGs reveal light-dependent output variations under illumination, as mentioned in the working mechanism section, making them self-powered photodetectors without using any power source. For example, the PENG based on $\mathrm{MAPbI}_{3}-\mathrm{PVDF}$ composite films displayed notable variations in piezoelectric output voltage and currents under simultaneous application of pressure and illuminations (Figure 6a) [131]. The device reported $\sim 42 \%$ decrement in the output voltage amplitudes, while $\sim 39 \%$ of increment in the current amplitudes upon illumination compared to dark mode values (Figure $6 b, c)$. According to the authors, this was due to the rapid generation of electrons and holes in photoactive $\mathrm{MAPbI}_{3}$ material under light, resulting in increasing photocurrent. The electron cloud enhances the local electromagnetic field within the composite film. It interrupts the orientation of the dipole, resulting in a lower piezoelectric output voltage than dark mode. However, there has been no clear study on this subject. Further experimental studies are needed to corroborate these understandings. The light-sensitive piezoelectric response 
of the nanogenerator unambiguously suggests that the nanogenerator can be operated as a flexible SPD irrespective of the working mechanism. The reported PENG based on different organic $\mathrm{HP}\left(\mathrm{FAPbBr}_{3}\right)$ showed similar light-induced variations in piezoelectric output upon light on/off process, suggesting the potential of the device to recognize the ambient light in a self-powered way [132]. On the other hand, the HP-based TENGs also exhibit a light-stimulated response upon illumination without the external power unit because of the combined triboelectric effect and photovoltaic properties of perovskite materials, supporting them as SPDs $[52,54,80,86]$. For example, the TENG based on inorganic $\mathrm{HP}$ material $\left(\mathrm{CsPbB}_{3}\right)$ was reported to display a light-reliant triboelectric response upon illumination while operating TENG [86]. As shown in Figure 6d, perovskite/FTO-glass and carbon/Ag-tape in fabricated TENG (i.e., FTO/CsPbBr $/$ / Carbon/Ag) act as triboelectric positive and negative materials, respectively. When the TENG was operated in vertical contact separation mode in a dark state, a very low output current of $\sim 0.3 \mu \mathrm{A}$ was obtained. It remarkably increased to $\sim 270 \mu \mathrm{A}$ when TENG was simultaneously illuminated. According to the author's hypothesis, when the carbon surface comes in contact with the perovskite surface during the TENG operation, a band bending takes place in upward direction at the surface of perovskite due to mismatch of work functions of both carbon $(4.81 \mathrm{eV})$ and $\mathrm{CsPbBr}_{3}(3.87 \mathrm{eV})$ materials. Further, when the TENG is illuminated during its operation, numerous electron-hole pairs are generated in the perovskite and a depletion region having an upward direction of build-in electric field is generated at the interface of carbon and perovskite during complete contact mode. The formed electron-hole pairs will be separated driven by the internal electric field, where the generated electrons would be collected by the FTO electrode, and the remaining holes would be moved onto the perovskite surface. The separation of photo-generated charges results a positive short-circuit photocurrent that imposes on triboelectric current and photo voltages upon contacting, thus leading to a significant increment in short-circuit current. However, light-induced outputs will disappear as the Schottky barrier disappears upon separation. On the other hand, no such light-dependent changes were observed for the device with a dielectric layer (PVDF) (i.e., FTO/perovskite/ / PVDF/Ag). This is because electrically insulating PVDF polymer cannot extract the photogenerated charge carriers despite the accumulation of a huge number of electron-hole pairs in the perovskite layer upon illumination due to mismatch of work function. It causes the rapid recombination of electrons and holes, providing constant outputs. Therefore, results suggest that the light-stimulated triboelectric output can operate the device as a self-powered light detector. Especially, the light sensitivity of the HP-based nanogenerators can be significantly enhanced by engineering the structure of the device; and to the end, many TENG-based SPDs were reported using a variety of HP materials $[52,54,80]$. Li et al. verified such a light behavior in other types of $\mathrm{HP}\left(\mathrm{MAPbI}_{3}\right)$-based TENG having the structure of $\mathrm{FTO} / \mathrm{TiO}_{2} / \mathrm{MAPbI}_{3} / / \mathrm{PTFE} / \mathrm{Cu}$. TENG was fabricated using light-active $\mathrm{MAPbI}_{3}$ and was spin-coated onto the $\mathrm{TiO}_{2}$ containing FTO-glass [52]. The compact $\mathrm{TiO}_{2}$ was used as a hole blocking layer, and the mesoporous $\mathrm{TiO}_{2}$ was used as a scaffold cum charge transport layer. $\mathrm{MAPbI}_{3}$ was used as a triboelectric layer and as a light-sensitive layer to enhance the triboelectric performance under illumination owing to the combined properties of photoelectric and triboelectric effects. Additionally, the TENG holds $\mathrm{Cu}$ deposited polytetrafluoroethylene (PTFE) film as a counter triboelectric part. The FTO and the copper layers were used as current collectors. When the TENG was operated under light, the triboelectric output voltage (peak-to-peak) amplitudes were rapidly raised by approximately $11 \%(\sim 17 \mathrm{~V})$ than the dark-state measurement value ( 15.3 V) (Figure 6e). The obtained light-reliant output performance from the device was mainly because of light-stimulated variations in surface charge density upon friction, as shown in Figure $6 f$. According to the authors, when the pressure was applied vertically on TENG, the contact electrification between PTFE and perovskite surface generated the surface charges with opposite polarity on both surfaces. Simultaneously, the electron-hole pairs were generated in the perovskite layer when the TENG was illuminated. Moreover, the excited electrons were readily extracted into $\mathrm{TiO}_{2}$ by leaving the holes behind on the surface. It amplified 
the triboelectric positive charge on the perovskite surface due to positive holes. Therefore, it resulted in enriched surface potential. The increment in surface potential increases the potential difference between both the electrodes; it amplified the output performance under the state of light. However, the output voltage of TENG notably decreased by approximately $37.5 \%(\sim 5 \mathrm{~V})$ immediately after the illumination (i.e., at one sun illumination, $100 \mathrm{~mW} / \mathrm{cm}^{2}$ ) than the dark-state value (Figure $6 \mathrm{~h}$ ) when the copper was used as a triboelectric friction layer in the same device structure as a replacement for PTFE film, as shown in Figure $6 \mathrm{~g}$ [80]. Furthermore, the device displayed a responsivity of $7.5 \mathrm{~V} / \mathrm{W}$ with a quick response time of $<80 \mathrm{~ms}$ (Figure 6i). It also showed good light on/off switching property, supporting the device as a self-powered photodetector for a wide range of light detection from UV to visible regions. Authors examined the surface charge density of perovskite film to clearly elucidate the light-controlled behavior of TENG using the scanning Kelvin probe microscope (SKPM) technique after rubbing it with friction film before and after light-illuminations at an applied bias voltage of $3 \mathrm{~V}$. A positive surface potential of $220 \mathrm{mV}$ was obtained on perovskite surface before rubbing it with PTFE film. It was increased to $580 \mathrm{mV}$ after $5 \mathrm{~min}$ of rubbing it with PTFE because of triboelectrification. Consequently, when the $\mathrm{MAPbI}_{3}$ was illuminated at an intensity of $70 \mathrm{~mW} / \mathrm{cm}^{2}$, the surface potential significantly increased to $730 \mathrm{mV}$ owing to the rapid movement of photogenerated holes onto the surface as explained in the earlier working mechanism [52]. However, when the sample was rubbed with a metal electrode; the surface charge density on $\mathrm{MAPbI}_{3}$ readily reduced under the exposure to light, leading to the decrement of TENG output for $\mathrm{Cu}$-friction or Al-friction layer [52,80]. Additionally, the photoconductivity of perovskite film, which increased rapidly under illumination, was another factor for reducing the device output. It was clearly explained by the authors using the AFM study. Therefore, the authors believe that the altered triboelectric surface charge and photoconductivity play a vital role in the working operation of the TENG as SPD. Moreover, another group has reported the robust, broad range (from UV to Visible region) SPD by engineering the device structure and perovskite material through halide ion tuning [54]. The authors used various configurations to construct TENGs covering $\mathrm{MAPbI}_{\mathrm{x}} \mathrm{Cl}_{3-\mathrm{x}}$ perovskite as a photoactive material and explored their output performances upon dark and illuminations owing to the combined triboelectric and photovoltaic effects of used perovskite. Among many, the TENG comprising the structure of $\mathrm{FTO} / \mathrm{TiO}_{2} / \mathrm{MAPbI}_{\mathrm{x}} \mathrm{Cl}_{3-\mathrm{x}} /$ pentacene//PTFE/Al revealed higher triboelectric performance under dark and larger voltage change (55.7\%) upon one sun illumination $\left(100 \mathrm{~mW} / \mathrm{cm}^{2}\right)$. The voltage in the dark-state increased from 14.64 to $22.80 \mathrm{~V}$ upon illumination due to the presence of both electron transport layer $\left(\mathrm{TiO}_{2}\right)$ and hole transport layer (pentacene) in TENG, which help to increase the charge density on the perovskite surface under illumination, as mentioned above. The increase in output of TENG with increasing light intensity $\left(0.01-100 \mathrm{~mW} / \mathrm{cm}^{2}\right)$ promoted the device to operate as an SPD, consisting of a high photo-responsivity of $119.3 \mathrm{~V} / \mathrm{W}$. Furthermore, the SPD revealed a wavelength-dependent response, making the device suitable for broad range light detection - from ultraviolet to visible region. The essential parameters including sensitivity values of the HP-nanogenerator based photodetectors are summarized in Table 1. These results suggest that the HP-based nanogenerator-a PENG or a TENG—can successfully function as a self-powered photodetector without the need for a battery or an external power supply. 


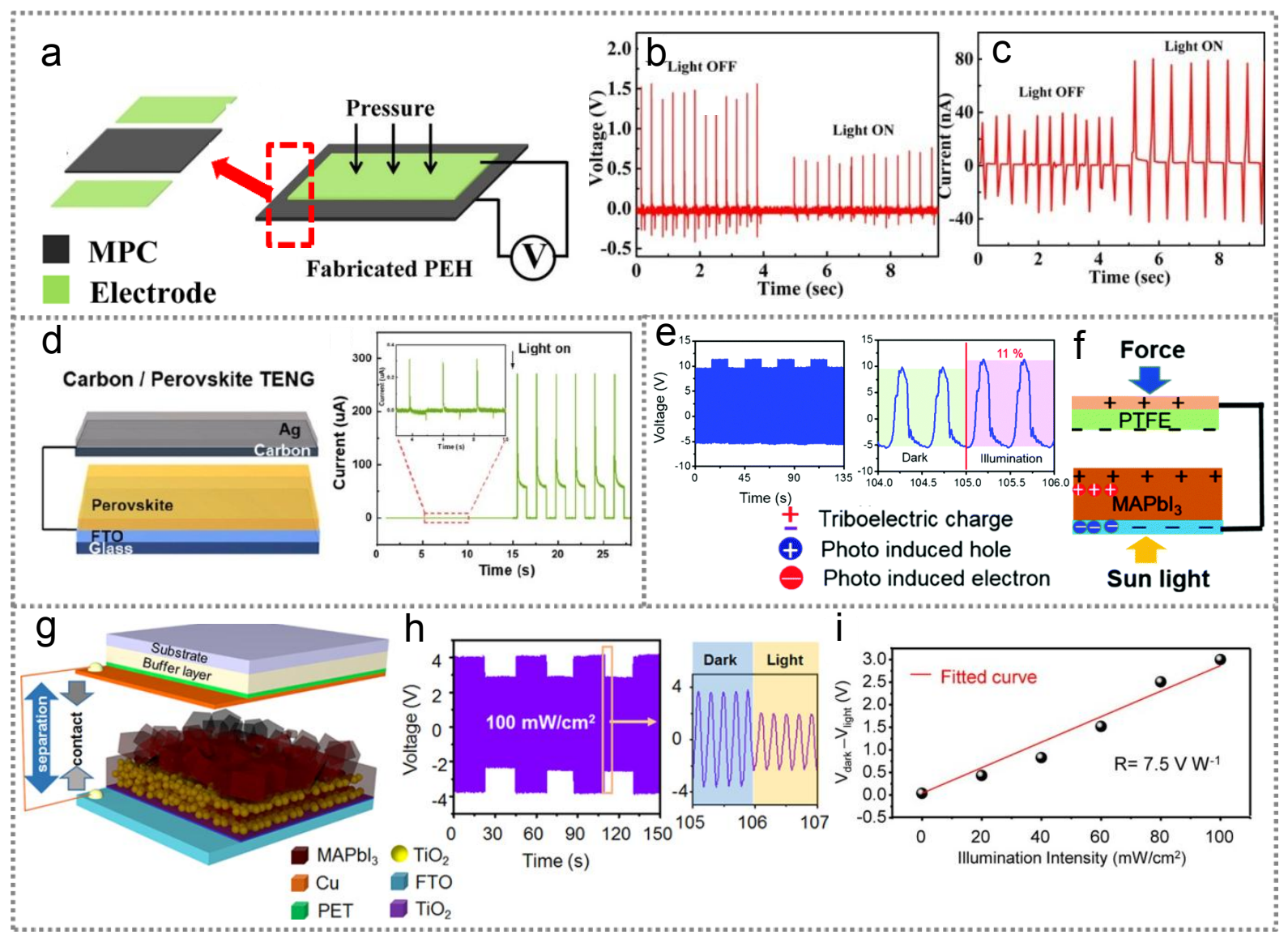

Figure 6. (a) Schematic image of $\mathrm{MAPbI}_{3}-\mathrm{PVDF}$ composite-based PENG, (b,c) corresponding light-controlled piezoelectric outputs. (a-c) Reprinted with permission from ref. [131], Copyright 2018, American Chemical Society. (d) Schematic image of $\mathrm{CsPbBr}_{3}$-based TENG and its light-controlled triboelectric output current. Reprinted with permission from ref. [86], Copyright 2020, Elsevier. (e) Light dependent output voltage of $\mathrm{MAPbI}_{3}$-based TENG, (f) corresponding TENG working mechanism under light and pressure. (e,f) Reprinted with permission from ref. [52], Copyright 2016, The Royal Society of Chemistry. (g) Schematic diagram of $\mathrm{MAPbI}_{3}$-based TENG, (h) corresponding light-controlled output voltage, and (i) light intensity dependent change in output voltage. (g-i) Reprinted with permission from ref. [80], Copyright 2015, American Chemical Society.

Table 1. Comparison of sensitivity values and change in output parameters of the HP-nanogenerator based sensors.

\begin{tabular}{|c|c|c|c|c|c|}
\hline Active Material & Device Structure & $\begin{array}{c}\text { Mode of } \\
\text { Operation }\end{array}$ & Sensor Type & $\begin{array}{c}\text { Sensitivity/ } \\
\text { Change in Output }\end{array}$ & Ref. \\
\hline $\mathrm{MAPbI}_{3}-\mathrm{PVDF}$ & $\mathrm{Ni}-\mathrm{MAPbI}_{3}-\mathrm{PVDF}-\mathrm{Cu}$ & PyENG & Temperature & $0.41 \mathrm{pA} / \mathrm{K}$ & {$[48]$} \\
\hline $\mathrm{MAPbBr}_{3}-\mathrm{PVDF}$ & $\mathrm{Ni}-\mathrm{NF} / \mathrm{MAPbBr} 3-\mathrm{PVDF} / \mathrm{Cu}-\mathrm{NF}$ & PENG & $\begin{array}{l}\text { Pressure and } \\
\text { acoustic }\end{array}$ & $\begin{array}{c}0.1 \mathrm{~V} / \mathrm{Pa} \text { and } \\
13.8 \mathrm{~V} / \mathrm{Pa}\end{array}$ & {$[50]$} \\
\hline $\mathrm{MASnI}_{3}$ & $\mathrm{PI} / \mathrm{Au} / \mathrm{MASnI}_{3} / \mathrm{PDMS} / \mathrm{ITO} / \mathrm{PET}$ & PENG & Pressure & $\sim 6.3 \mathrm{~V} / \mathrm{MPa}$ & [117] \\
\hline MASnBr 3 -PDMS & $\begin{array}{l}\mathrm{PI} / \mathrm{Au} / \mathrm{MASnBr}_{3}^{-} \\
\mathrm{PDMS} / \mathrm{ITO} / \mathrm{PET}\end{array}$ & PENG & $\begin{array}{l}\text { Pressure and } \\
\text { Physiological }\end{array}$ & $\sim 57 \mathrm{~V} / \mathrm{MPa}$ & {$[45]$} \\
\hline $\mathrm{TMCM}_{2} \mathrm{SnCl}_{6}$ & $\mathrm{Cu} / \mathrm{TMCM}_{2} \mathrm{SnCl}_{6}-\mathrm{PDMS} / \mathrm{Cu}$ & PENG & Pressure & $\sim 19.2 \mathrm{~V} / \mathrm{N}$ & [120] \\
\hline $4 \mathrm{Cl}-\mathrm{MAPbI}_{3}$ & $\begin{array}{c}\mathrm{PI} / \mathrm{Au} / 4 \mathrm{Cl}- \\
\mathrm{MAPbI}_{3} / \mathrm{PDMS} / \mathrm{ITO} / \mathrm{PET}\end{array}$ & PENG & Physiological & - & [66] \\
\hline $\mathrm{CsPbBr}_{3}$ & $\mathrm{PET} / \mathrm{ITO} / \mathrm{CsPbBr}_{3} / \mathrm{PDMS} / \mathrm{ITO} / \mathrm{PET}$ & PENG & Physiological & - & [79] \\
\hline $\mathrm{MAPbI}_{3}-\mathrm{PDMS}$ & $\mathrm{Cu} / \mathrm{MAPbI}_{3} / \mathrm{Pt}$ & PENG & Physiological & - & [121] \\
\hline
\end{tabular}


Table 1. Cont.

\begin{tabular}{|c|c|c|c|c|c|}
\hline Active Material & Device Structure & $\begin{array}{c}\text { Mode of } \\
\text { Operation }\end{array}$ & Sensor Type & $\begin{array}{c}\text { Sensitivity/ } \\
\text { Change in Output }\end{array}$ & Ref. \\
\hline $\mathrm{MAPbI}_{3}-\mathrm{PVDF}$ & $\begin{array}{l}\mathrm{PET} / \mathrm{ITO} / \mathrm{MAPbI}_{3}- \\
\mathrm{PVDF} / \mathrm{Carbon} \text { tape }\end{array}$ & PENG & photodetector & $\begin{array}{c}42 \% \text { change } \\
\left(\mathrm{V}_{\text {dark }}=1.4 \mathrm{~V} \text { and }\right. \\
\left.\mathrm{V}_{\text {light }}=0.8 \mathrm{~V}\right)\end{array}$ & [131] \\
\hline $\mathrm{FAPbBr}_{3}-\mathrm{PVDF}$ & $\mathrm{FTO} / \mathrm{FAPbBr}_{3}-\mathrm{PVDF} / \mathrm{Ag}$ & PENG & Photodetector & $\begin{array}{c}38 \% \text { change } \\
\left(\mathrm{V}_{\text {dark }}=26.2 \mathrm{~V} \text { and }\right. \\
\left.\mathrm{V}_{\text {light }}=16.1 \mathrm{~V}\right)\end{array}$ & [127] \\
\hline $\mathrm{CsPbBr}_{3}$ & $\mathrm{FTO} / \mathrm{CsPbBr}_{3} / /$ Carbon/Ag & TENG & Photodetector & $\begin{array}{c}900 \% \text { change } \\
\left(\mathrm{I}_{\text {dark }}=0.3 \mu \mathrm{A} \text { and }\right. \\
\mathrm{I}_{\text {light }}=270 \mu \mathrm{A} \text { in } \\
\text { one sun })\end{array}$ & [90] \\
\hline $\mathrm{MAPbI}_{3}$ & $\mathrm{FTO} / \mathrm{TiO}_{2} / \mathrm{MAPbI}_{3} / / \mathrm{PTFE} / \mathrm{Cu}$ & TENG & Photodetector & $\begin{array}{c}11 \% \text { change } \\
\left(\mathrm{V}_{\text {dark }}=15.3 \mathrm{~V} \text { and }\right. \\
\mathrm{V}_{\text {light }}=17 \mathrm{~V} \text { in one } \\
\text { sun })\end{array}$ & [52] \\
\hline $\mathrm{MAPbI}_{3}$ & $\mathrm{FTO} / \mathrm{TiO}_{2} / \mathrm{MAPbI}_{3} / / \mathrm{Cu}$ & TENG & Photodetector & $7.5 \mathrm{~V} / \mathrm{W}$ & [80] \\
\hline $\mathrm{MAPbI}_{\mathrm{x}} \mathrm{Cl}_{3-\mathrm{x}}$ & $\begin{array}{c}\mathrm{FTO} / \mathrm{TiO}_{2} / \mathrm{MAPbI}_{\mathrm{x}} \mathrm{Cl}_{3-\mathrm{x} /} \\
\text { pentacene//PTFE/Al }\end{array}$ & TENG & Photodetector & $\begin{array}{c}119.3 \mathrm{~V} / \mathrm{W} \text { and } \\
55.7 \% \text { change } \\
\left(\mathrm{V}_{\text {dark }}=14.64 \mathrm{~V}\right. \\
\text { and } \mathrm{V}_{\text {light }}=22.8 \mathrm{~V} \\
\text { in one sun })\end{array}$ & [54] \\
\hline $\mathrm{MAPbI}_{3}$ & Glass / AAA $/ \mathrm{MAPbI}_{3} / \mathrm{SiO}_{2} / \mathrm{AAA}$ & PENG & $\begin{array}{l}\text { Bimodal (Pressure } \\
\text { and light) }\end{array}$ & $8.43 \mathrm{mV} / \mathrm{kPa}$ & [55] \\
\hline $\mathrm{MAPbI}_{3}-\mathrm{PVDF}$ & $\mathrm{PET} / \mathrm{MAPbI}_{3}-\mathrm{PVDF} / \mathrm{Au}-\mathrm{IDEs}$ & PENG & $\begin{array}{c}\text { Bimodal } \\
\text { (Pressure and } \\
\text { light) }\end{array}$ & $\begin{array}{l}0.107 \mathrm{~V} / \mathrm{kPa} \text { and } \\
129.2 \mathrm{~V} / \mathrm{mW}\end{array}$ & {$[51]$} \\
\hline $\mathrm{CsPbBr}_{3}$ & PET/PEDOT:PSS/CsPbBr $3 / \mathrm{Au}$ & TENG & $\begin{array}{l}\text { Bimodal (Pressure } \\
\text { and UV light) }\end{array}$ & $\begin{array}{c}\sim 5.1 \mathrm{~V} . \mathrm{cm}^{-2} / \mathrm{N} \\
\text { and } 145 \% \text { change } \\
\left(\mathrm{V}_{\text {dark }}=2.27 \mathrm{~V} \text { and }\right. \\
\mathrm{V}_{\text {light }}=5.56 \mathrm{~V} \\
\text { under } 100 \\
\left.\mathrm{~mW} / \mathrm{cm}^{2}\right)\end{array}$ & [53] \\
\hline
\end{tabular}

\subsection{Halide Perouskite Nanogenerator-Based Self-Powered Bimodal Sensors}

Recently, several emerging sensor technologies have been developed to detect singlemode external stimuli, such as light, pressure, temperature, and humidity [133-136]. However, emerging a flexible single sensor unit capable of sensing multiple signals simultaneously is of great significance. Stacking or incorporating single-mode sensor units to build a multimode sensor can be constrained by the signal-interference of different sensor units and disparities in their mechanical properties [137,138]. Therefore, it is of prodigious importance to fabricate a flexible single-structure bi/multifunctional sensor capable of perceiving various external signals using flexible materials in simple and low-cost methods. Hence, HP materials are the potential entrants to develop bi/multifunctional sensor devices in facile ways owing to the combined properties such as optoelectronic, piezo/ferroelectric, pyroelectric, and thermoelectric properties [37,71,139-141]. Eom et al. constructed the first HP material-based self-powered bimodal sensor (i.e., pressure cum light sensor) covering the PENG structure of glass / AAA $/ \mathrm{MAPbI}_{3} / \mathrm{SiO}_{2} / \mathrm{AAA}$ (Figure 7a) (Here, AAA is $\mathrm{AZO}-\mathrm{Au}-\mathrm{AZO}$ multilayer) [55]. The $\mathrm{MAPbI}_{3}$ perovskite thin film was prepared using the chemical vapor deposition (CVD) technique to study its long-term air-stability. The sputtered thin $\mathrm{SiO}_{2}$ layer helped to prevent the electrical short-circuit of the device. The nanogenerator poled at $60 \mathrm{kV} / \mathrm{cm}$ exhibited a linearly increasing trend in piezoelectric output with increasing applied pressure from 5 to $70 \mathrm{kPa}$ and obtained the pressure sensitivity 
value of $\sim 8.43 \mathrm{mV} / \mathrm{kPa}$ (see Figure $7 \mathrm{~b}$ ). The device was simultaneously exposed to light while applying strain $(30 \mathrm{kPa})$ to explore the bimodal sensing (pressure and light) features of the nanogenerator. It was observed that the device displayed a notable dependency on the intensity of focused light (Figure 7c), signifying the ability of the device to identify different intensities of light in a self-powered way. Another research group has also verified the multimode sensing (i.e., light and pressure signals sensing) ability of inorganic HP material-based TENG [53]. The reported light-stimulated e-skin-based TENG has the structure of PET/PEDOT: PSS/CsPbBr 3 / Au. It was operated based on combined photoelectric and triboelectric effects of $\mathrm{CsPbBr}$ material, as illustrated in Figure 7d. Under repeated vertical contact-separations of the e-skin by finger tapping, the triboelectric output was notably increased with increasing applied force, indicating the low-level pressure sensing ability of the nanogenerator in a self-powered way (Figure 7e). Additionally, authors have used the same device to identify the various intensities of light by illuminating the e-skin using UV-light $(\sim 365 \mathrm{~nm})$ for $8 \mathrm{~s}$ at an applied force of $0.3 \mathrm{~N}$. They observed the light-dependent responsivity of the device, as shown in Figure 7f. Furthermore, it is believed that the e-skin can be successfully implemented as a memristor to comprehend the human-machine interfaces in emerging artificial intelligence systems. Improving the long-term stability of HPs is imperative and remains a key challenge irrespective of their great potentiality as active sensing materials. Recently our group has found the tremendous bimodal sensing application of HP-polymer composite by emerging long-term stable single-structure device using a simple and cost-effective approach [51]. The flexible single-structure multifunctional device capable of mechanical energy scavenging and simultaneous sensing of multi-physical signals-light and pressure - was constructed using spin-coated $\mathrm{MAPbI}_{3}-\mathrm{PVDF}$ composite film onto the flexible plastic substrate followed by sputtering of gold-inter digitated electrodes (gold-IDE) (Figure 7g). The reported nanogenerator was successfully operated as an energy harvester. Furthermore, it was simultaneously operated as a self-powered light and pressure sensors in both piezoelectric and triboelectric modes of operation owing to the combined photovoltaic and piezo/triboelectric effects of the active composite film. It was observed that the piezoelectric output of the device under a dark state increased linearly with increasing applied pressure. It displayed high output voltage of $\sim 33.6 \mathrm{~V}$ at an applied pressure of $300 \mathrm{kPa}$, making the device a potential candidate to be used as a self-powered pressure sensor with excellent pressure sensitivity of $0.107 \mathrm{~V} / \mathrm{kPa}$ (Figure $7 \mathrm{~h}$ ). The output in dark-state for PENG mode (double electrode mode-SPPD) decreased significantly during illumination with increasing light intensity within $0.06-3.23 \mathrm{~mW} / \mathrm{cm}^{2}$ range. It exhibited a large voltage change $(\Delta \mathrm{V})$ of $\sim 90 \%$ at intensity of $3.23 \mathrm{~mW} / \mathrm{cm}^{2}$ (Figure $7 \mathrm{i}$ ). On the contrary, the output obtained in dark-state for TENG mode (single electrode mode-STPD) notably increased with increasing illumination intensity. It showed a $\Delta \mathrm{V}$ of $\sim 51 \%$ at a high intensity of $3.23 \mathrm{~mW} / \mathrm{cm}^{2}$. The nanogenerator in PENG-SPPD and TENG-STPD modes showed notable photo-responsivity values of 129.2 and $79.7 \mathrm{~V} / \mathrm{mW}$ at the lowest light intensity of $0.06 \mathrm{~mW} / \mathrm{cm}^{2}$, respectively. These results indicate outstanding light-detecting ability in both piezoelectric and triboelectric modes of nanogenerator with a small quantity of $\mathrm{MAPbI}_{3}(25 \mathrm{vol} \%)$ in PVDF matrix. We conducted the PFM study for $\mathrm{MAPbI}_{3}-\mathrm{PVDF}$ composite film under dark and illuminations (optical microscope light) to study the working principle of nanogenerator as a self-powered photodetector. We observed the decrement $(\sim 13 \%)$ in piezoelectric coefficient $\left(\mathrm{d}_{33}\right)$ from $\sim 14.58$ (dark-state) to $\sim 12.68 \mathrm{pm} / \mathrm{V}$ under illumination owing to the rapid generation of photogenerated charge carriers that increase the leaky nature of the film. We believe that detailed research is required to evidently portray the light-stimulated piezo- and triboelectric effects in light-active HP materials. Additionally, the nanogenerator demonstrated good light on-off switching properties, outstanding mechanical endurance, and significantly good air stability (more than six months). The authors also achieved the real-time applications of the reported nanogenerator as a self-powered bimodal sensor (pressure cum light sensor) by exposing the device to real environs besides the laboratory measurements (Figure 7j). It was observed that the nanogenerator displayed a notable voltage change $(\Delta V)$ with different light environs and 
times of a given day, indicating its excellent light perceiving ability (Figure 7k). Therefore, it was established that the pressure-dependent and the light-controlled response of the nanogenerator allow the device to be operated as a pressure and photodetectors (i.e., bimodal sensor), respectively, in a self-powered way. Furthermore, the nanogenerator performance in both PENG and TENG modes was significantly altered by the external matter to exert pressure due to the contact electrification effect, suggesting the device's potential for e-skin, IoT, and AI electronic device applications. The bimodal sensors based on HP materials have been used to detect both pressure and lights simultaneously or individually owing to the combined piezo/triboelectric and photovoltaic properties. The essential parameters including sensitivity values of the HP-based bimodal sensors are summarized in Table 1. However, in case of bi-modal/multi-mode sensors, it is very important to eliminate the interference effect on the multi-mode signals during its operation. In case of HP-based bimodal sensors, light was detected in terms of change in piezoelectric or triboelectric output performance of the nanogenerators in which light-induced pressure-dependent piezoelectric or triboelectric output is clearly observed. However, both PENG and TENG under only illumination generate very lower or negligible outputs when compared with the pressure-dependent outputs. Therefore, when the device is operated as a bimodal sensor there may be a negligible interference effect in the output performance of sensor. In addition, another possibility to eliminate the interference effect is to fabricate the device with number of arrays and measure the output performances individually using both the sources. Later the device should be exposed to multiple sources and characterize the performance. When the device was applied as a sensor in real environments the variations in level of output signals will decide the type and amount of input signal. However, more efforts are needed in terms of technological or electrical aspects to minimize such effects in bimodal sensors. The reported bimodal sensors based on HP materials until today have been used to detect only pressure and lights. However, studying other properties of HPs, such as pyroelectric or thermoelectric properties together with optoelectronic properties, may provide a way to develop temperature cum light bimodal sensors. Additionally, extending the research to combine three or more properties may still pave the way to fabricate multimode sensors. For example, our group reported HP material-based TPS-fusion energy harvester capable of harvesting multiple energy sources such as thermal, mechanical, and light energies owing to the multiple properties of comprised $\mathrm{MAPbI}_{3}$ material [118]. The flexible TPS-nanogenerator was fabricated on a flexible plastic substrate by spin-coating of $\mathrm{MAPbI}_{3}$ followed by sputtering of $\mathrm{Cu}_{2} \mathrm{O}, \mathrm{ZnO}$, and ITO thin film IDEs and operated individually under three different energy sources-temperature, pressure, and solar light. The TPS-nanogenerator as a thermoelectric nanogenerator demonstrated the high thermoelectric output voltage of $0.12 \mathrm{~V}$ at $\Delta \mathrm{T}$ of $14.9^{\circ} \mathrm{C}$. The nanogenerator as a piezoelectric nanogenerator achieved the piezoelectric voltage of $\sim 1.47 \mathrm{~V}$ under periodically applied pressure of $0.2 \mathrm{MPa}$. However, the same TPS device as a solar cell generated an output voltage of $0.77 \mathrm{~V}$ under one sun illumination. Therefore, it is suggested that a thorough and simultaneous investigation of these three properties may develop a trimodal sensor (i.e., temperature, light, and pressure) using single material and single-structure device. Therefore, all the matter discussed above explicates the potential of HP or HP-polymer composite materials in emerging self-powered sensors without the need of any external power supply to perceive external stimuli, such as pressure, temperature, and light independently or simultaneously. 


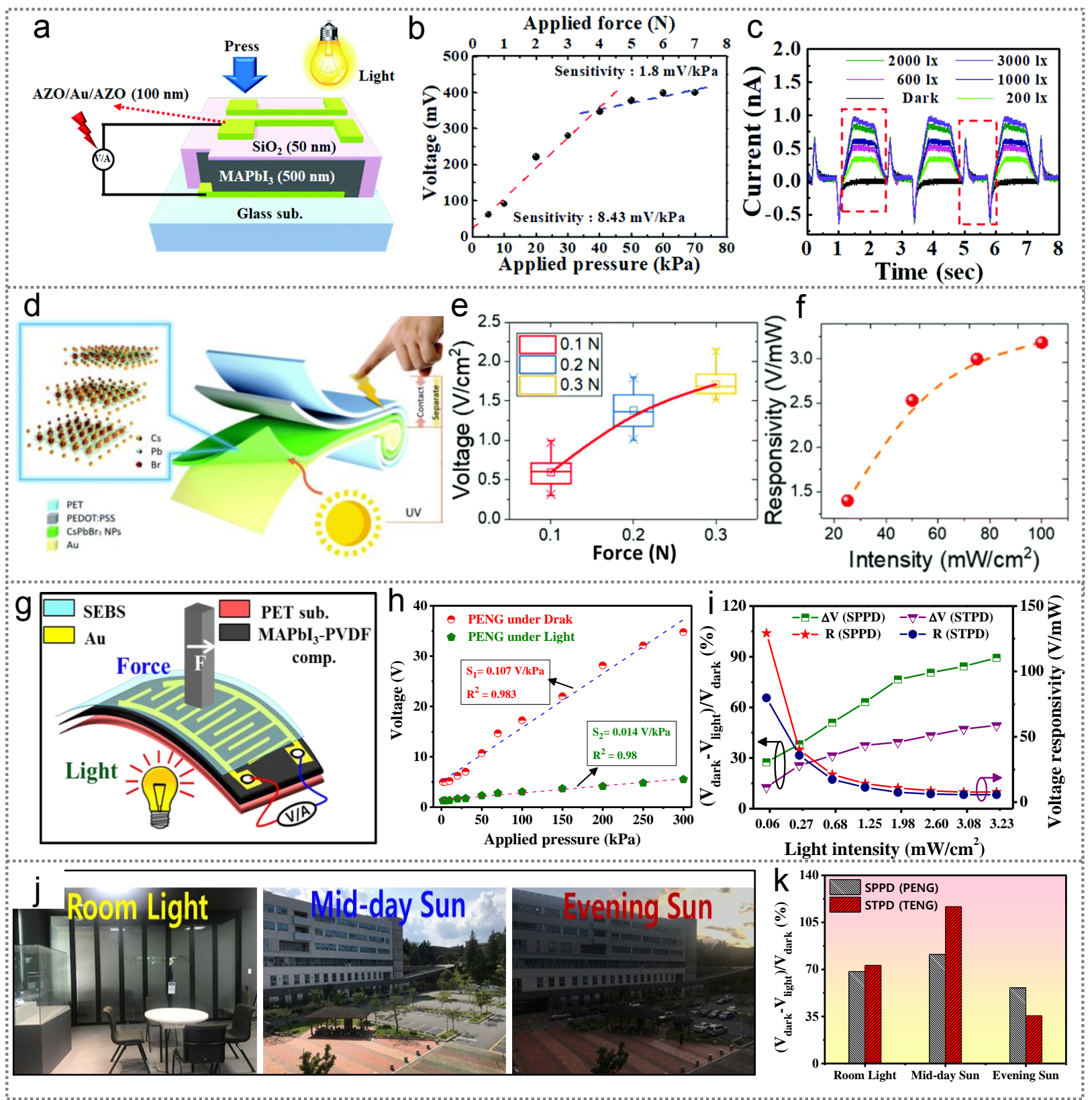

Figure 7. (a) Schematic illustration of $\mathrm{MAPbI}_{3}$ PENG-based bimodal sensor, (b) corresponding pressure dependent output voltages revealing the pressure sensitivity, and (c) light-controlled piezoelectric output current signals at an applied constant pressure of $30 \mathrm{kPa}$. (a-c) Reprinted with permission from ref. [55], Copyright 2018, The Royal Society of Chemistry. (d) Schematic representation of $\mathrm{CsPbBr}_{3}$ TENG-based bimodal sensor, (e) corresponding pressure dependent output voltage, and (f) responsivity of the device under various light intensities. (d-f) Reprinted with permission from ref. [53], Copyright 2021, The Royal Society of Chemistry. (g) Schematic sketch of MAPbI $_{3}$-PVDF-based bimodal sensor in PENG mode, (h) corresponding pressure sensitivity under dark and illumination, (i) corresponding voltage change and responsivity with respect to light intensities, (j) digital images of various light environments used for real-time operation of the device, and $(\mathbf{k})$ corresponding change in voltages. (g-k) Reprinted with permission from ref. [51], Copyright 2020, American Chemical Society.

\section{Conclusions, Outlooks, and Opportunities for Future Development}

HPs have been recognized as potential entrants in numerous research fields, including solar cells, LEDs, nanogenerators, and photodetectors. Among these, the nanogenerators are the recent application of HPs. They are highly sensitive to various external perturbations, such as pressure, temperature, and light. Hence, controlling the performance of a 
nanogenerator under external perturbations such as temperature and pressure predominantly enabled it to be used as a self-powered temperature or pressure sensor, respectively, without dependency on a battery. Additionally, illuminating nanogenerator significantly changes their output performance owing to the combined optoelectronic properties with piezoelectric property, or triboelectric effect. Such light-stimulated performances of nanogenerators prominently enabled them to be used as self-powered photodetectors to detect the type of light or quantity of light intensity. This progress report briefly discussed the structural, piezoelectric, pyroelectric, and triboelectric properties of nanogenerators of $\mathrm{HP}$ materials. After that, we discussed the working mechanisms of several nanogenerators, including PENG, TENG, and PyENG as self-powered pressure and temperature sensors. We discussed the operation of PENG and TENG, as self-powered photodetectors under light illumination and present the possible underlying mechanisms to deliver the light-controlled outputs. Thereafter, we provided an overview of the exploration of using $\mathrm{HP}$-based nanogenerators to sense various external factors such as pressure, temperature changes, and light. It is seen from the examples discussed above that when a nanogenerator is subjected to a controlled exposure to these external stimuli, continuous output voltage and current change linearly, which is a prerequisite for realizing reliable sensing systems. The recently emerging bimodal/multimodal sensing applications of HPs were also reviewed in addition to the single-mode sensor devices. We suggest the future projections of the research towards developing single-structure multimodal sensing devices.

Improving sensitivity, performance, and long-term stability of HPs is challenging despite many studies involving HPs as active sensing materials in self-powered nanogeneratorbased sensors. Therefore, we identify potential directions and opportunities for future research. Recently, numerous methods, such as surface passivation, compositional tuning, fine-doping, and polymer-composites, have been used to improve the air-stability of HP materials $[45,51,68]$. Furthermore, it has been demonstrated that the layered 2D-HPs have significantly good air and moisture stability than the 3D-HPs because of long-chain organic molecules [142,143]. Additionally, these 2D-HPs offer extremely high piezo/ferroelectric properties, helping to construct flexible, high-performance nanogenerators and reliable sensors with high sensitivity and stability [120]. Additionally, the development of eco-friendly HP material-based self-powered sensors has been highly prioritized to alleviate the environmental and human health risks. Additionally, extending the investigation to combine three or more properties may be helpful in developing trimodal/multimodal sensors to detect multiple physical signals simultaneously using a single-structure device without any signal interference. For example, our group has reported a single-structure TPS-fusion generator capable of harvesting multiple energy sources, such as thermal, mechanical, and light energies owing to the multiple properties of comprised $\mathrm{MAPbI}_{3}$ material [118]. Finally, the flexible and stretchable self-powered sensing systems have significant potential to be for e-skin and wearable electronic device applications in the future. In this regard, considerable research should be conducted on HP materials to develop self-powered stretchable multifunctional devices that can continuously operate in harsh environs.

Author Contributions: S.I.: Conceptualization, visualization, validation, formal analysis, resources, data curation, writing — original draft preparation, writing-review and editing, funding acquisition. V.J.: Conceptualization, validation, formal analysis, resources, data curation, writing—original draft preparation, writing-review and editing, supervision, project administration, funding acquisition. A.M.T.: Visualization, validation, formal analysis. S.-G.Y.: Conceptualization, validation, formal analysis, resources, data curation, writing —original draft preparation, writing — review and editing, supervision, project administration, funding acquisition. All authors have read and agreed to the published version of the manuscript.

Funding: This work was supported by a National Research Foundation of Korea (NRF) grant funded by the Korean government (MSIP) (NRF-2021R1A2B5B03002016, NRF-2021R1A6A1A03043682, NRF2021R1A2C1010797, NRF-2021R1I1A1A01060012).

Conflicts of Interest: The authors declare no conflict of interests. 


\section{References}

1. Shi, Q.; Dong, B.; He, T.; Sun, Z.; Zhu, J.; Zhang, Z.; Lee, C. Progress in wearable electronics/photonics-moving toward the era of artificial intelligence and internet of things. InfoMat 2020, 2, 1131-1162. [CrossRef]

2. Shen, T.; Li, F.; Zhang, Z.; Xu, L.; Qi, J. High-Performance broadband photodetector based on monolayer mos 2 hybridized with environment-friendly CuInSe 2 quantum dots. ACS Appl. Mater. Interfaces 2020, 12, 54927-54935. [CrossRef]

3. Xu, F.; Li, X.; Shi, Y.; Li, L.; Wang, W.; He, L.; Liu, R. Recent developments for flexible pressure sensors: A review. Micromachines 2018, 9, 580. [CrossRef]

4. Kuzubasoglu, B.A.; Bahadir, S.K. Flexible temperature sensors: A review. Sens. Actuators A Phys. 2020, 315, 112282. [CrossRef]

5. Wen, F.; He, T.; Liu, H.; Chen, H.-Y.; Zhang, T.; Lee, C. Advances in chemical sensing technology for enabling the next-generation self-sustainable integrated wearable system in the IoT era. Nano Energy 2020, 78, 105155. [CrossRef]

6. Liu, Y.; Dong, X.; Chen, P. Biological and chemical sensors based on graphene materials. Chem. Soc. Rev. 2012, 41, 2283-2307. [CrossRef]

7. Han, X.; Lu, L.; Zheng, Y.; Feng, X.; Li, Z.; Li, J.; Ouyang, M. A review on the key issues of the lithium ion battery degradation among the whole life cycle. eTransportation 2019, 1, 100005. [CrossRef]

8. Chen, Y.; Kang, Y.; Zhao, Y.; Wang, L.; Liu, J.; Li, Y.; Liang, Z.; He, X.; Li, X.; Tavajohi, N.; et al. A review of lithium-ion battery safety concerns: The issues, strategies, and testing standards. J. Energy Chem. 2021, 59, 83-99. [CrossRef]

9. Li, Z.; Zheng, Q.; Wang, Z.L.; Li, Z. Nanogenerator-based self-powered sensors for wearable and implantable electronics. Research 2020, 2020, 8710686. [CrossRef] [PubMed]

10. Anaya, D.V.; He, T.; Lee, C.; Yuce, M.R. Self-powered eye motion sensor based on triboelectric interaction and near-field electrostatic induction for wearable assistive technologies. Nano Energy 2020, 72, 104675. [CrossRef]

11. Zhu, M.; Yi, Z.; Yang, B.; Lee, C. Making use of nanoenergy from human-Nanogenerator and self-powered sensor enabled sustainable wireless IoT sensory systems. Nanotoday 2021, 36, 101016. [CrossRef]

12. Zhou, H.; Zhang, Y.; Qiu, Y.; Wu, H.; Qin, W.; Liao, Y.; Yu, Q.; Cheng, H. Stretchable piezoelectric energy harvesters and self-powered sensors for wearable and implantable devices. Biosens. Bioelectron. 2020, 168, 112569. [CrossRef]

13. Zhou, Y.; Shen, M.; Cui, X.; Shao, Y.; Li, L.; Zhang, Y. Triboelectric nanogenerator based self-powered sensor for artificial intelligence. Nano Energy 2021, 84, 105887. [CrossRef]

14. He, T.; Guo, X.; Lee, C. Flourishing energy harvesters for future body sensor network: From single to multiple energy sources. IScience 2021, 24, 101934. [CrossRef]

15. Jin, H.; Shibli, A.R.Y.; Hossam, H. Advanced materials for health monitoring with skin based wearable devices. Adv. Healthc. Mater. 2017, 6, 1700024. [CrossRef]

16. Ding, Y.; Yang, J.; Tolle, C.R.; Zhu, Z. A highly stretchable strain sensor based on electrospun carbon nanofibers for human, Motion Monitoring. RSC Adv. 2016, 6, 79114-79120. [CrossRef]

17. Zang, Y.; Zhang, F.; Di, C.-a.; Zhu, D. Advances of flexible pressure sensors toward artificial intelligence and health care, Applications. Mater. Horiz. 2015, 2, 140-156. [CrossRef]

18. Wang, X.; Dong, L.; Zhang, H.; Yu, R.; Pan, C.; Wang, Z.L. Recent progress in electronic skin. Adv. Sci. 2015, 2, 1500169. [CrossRef]

19. Huang, Y.; Fang, D.; Wu, C.; Wang, W.; Guo, X.; Liu, P. A flexible touch-pressure sensor array with wireless transmission system for robotic skin. Rev. Sci. Instrum. 2016, 87, 065007. [CrossRef]

20. Dong, B.; Yang, Y.; Shi, Q.; Xu, S.; Sun, Z.; Zhu, S.; Zhang, Z.; Kwong, D.-L.; Zhou, G.; Ang, K.-W.; et al. Wearable triboelectric-human-machine interface (THMI) using robust nanophotonic readout. ACS Nano 2020, 14, 8915-8930. [CrossRef]

21. Garcia, C.; Trendafilova, I.; de Villoria, R.G.; del Rio, J.S. Self-powered pressure sensor based on the triboelectric effect and its analysis using dynamic mechanical analysis. Nano Energy 2018, 50, 401-409. [CrossRef]

22. Parida, K.; Bhavanasi, V.; Kumar, V.; Bendi, R.; Lee, P.S. Self-powered pressure sensor for ultra-wide range pressure detection. Nano Res. 2017, 10, 3557-3570. [CrossRef]

23. Korkmaz, S.; Kariper, I.A. Pyroelectric nanogenerators (PyNGs) in converting thermal energy into electrical energy: Fundamentals and current status. Nano Energy 2021, 84, 105888. [CrossRef]

24. Zhao, T.; Jiang, W.; Liu, H.; Niu, D.; Li, X.; Liu, W.; Li, X.; Chen, B.; Shi, Y.; Yin, L. An infrared-driven flexible pyroelectric generator for non-contact energy harvester. Nanoscale 2016, 8, 8111-8117. [CrossRef]

25. Liu, Y.; Chang, Y.; Sun, E.; Li, F.; Zhang, S.; Yang, B.; Sun, Y.; Wu, J.; Cao, W. Significantly enhanced energy-harvesting performance and superior fatigue-resistant behavior in $(001) \mathrm{c}$-textured $\mathrm{BaTiO}_{3}$-based lead-free piezoceramics. ACS Appl. Mater. Inter. 2018, 10, 31488-31497. [CrossRef]

26. Lee, H.; Kim, H.; Kim, D.Y.; Seo, Y. Pure piezoelectricity generation by a flexible nanogenerator based on lead zirconate titanate nanofibers. ACS Omega 2019, 4, 2610-2617. [CrossRef]

27. Johar, M.A.; Waseem, A.; Hassan, M.A.; Bagal, I.V.; Abdullah, A.; Ha, J.S.; Ryu, S.W. Highly durable piezoelectric nanogenerator by heteroepitaxy of $\mathrm{GaN}$ nanowires on $\mathrm{cu}$ foil for enhanced output using ambient actuation sources. Adv. Energy Mater. 2020, 10, 2002608. [CrossRef]

28. Le, A.T.; Ahmadipour, M.; Pung, S.-Y. A review on ZnO-based piezoelectric nanogenerators: Synthesis, characterization techniques, performance enhancement and applications. J. Alloys Compd. 2020, 844, 156172. [CrossRef] 
29. Choi, M.-J.; Eom, J.-H.; Shin, S.-H.; Nah, J.; Choi, J.-S.; Song, H.-A.; An, H.; Kim, H.Y.; Pammi, S.V.N.; Choi, G.; et al. Most facile synthesis of $\mathrm{Zn}-\mathrm{Al}: \mathrm{LDH}$ nanosheets at room temperature via environmentally friendly process and their high power generation by flexoelectricity. Mater. Today Energy 2018, 10, 254-263. [CrossRef]

30. Lu, L.; Ding, W.; Liu, J.; Yang, B. Flexible PVDF based piezoelectric nanogenerators. Nano Energy 2020, 78, 105251. [CrossRef]

31. Nguyen, T.M.T.; Ippili, S.; Eom, J.H.; Jella, V.; Tran, D.V.; Yoon, S.-G. Enhanced output performance of nanogenerator based on composite of poly vinyl fluoride (PVDF) and Zn:Al layered-double hydroxides (LDHs) nanosheets. Transact. Electr. Electron. Mater. 2018, 19, 403-411. [CrossRef]

32. Luo, Y.; Szafraniak, I.; Zakharov, N.D.; Nagarajan, V.; Steinhart, M.; Wehrspohn, R.B.; Wendorff, J.H.; Ramesh, R.; Alexe, M. Nanoshell tubes of ferroelectric lead zirconate titanate and barium titanate. Appl. Phys. Lett. 2003, 83, 440-442. [CrossRef]

33. Chen, X.; Xu, S.; Yao, N.; Xu, W.; Shi, Y. Potential measurement from a single lead ziroconate titanate nanofiber using a nanomanipulator. Appl. Phys. Lett. 2009, 94, 253113. [CrossRef]

34. Jiang, W.; Zhang, R.; Jiang, B.; Cao, W. Characterization of piezoelectric materials with large piezoelectric and electromechanical coupling coefficients. Ultrasonics 2003, 41, 55-63. [CrossRef]

35. Yoo, J.J.; Seo, G.; Chua, M.R.; Park, T.G.; Lu, Y.; Rotermund, F.; Kim, Y.K.; Moon, C.S.; Jeon, N.J.; Correa-Baena, J.P.; et al. Efficient perovskite solar cells via improved carrier management. Nature 2021, 590, 587-593. [CrossRef] [PubMed]

36. Wang, P.; Wu, Y.; Cai, B.; Ma, Q.; Zheng, X.; Zhang, W.-H. Solution-processable perovskite solar cells toward commercialization: Progress and challenges. Adv. Funct. Mater. 2019, 29, 1807661. [CrossRef]

37. Filip, M.R.; Eperon, G.E.; Snaith, H.J.; Giustino, F. Steric engineering of metal-halide perovskites with tunable optical band gaps. Nat. Commun. 2014, 5, 5757. [CrossRef] [PubMed]

38. Yang, Z.; Yu, Z.; Wei, H.; Xiao, X.; Ni, Z.; Chen, B.; Deng, Y.; Habisreutinger, S.N.; Chen, X.; Wang, K.; et al. Enhancing electron diffusion length in narrow-bandgap perovskites for efficient monolithic perovskite tandem solar cells. Nat. Commun. 2019, 10, 4498. [CrossRef]

39. Wehrenfennig, C.; Eperon, G.E.; Johnston, M.B.; Snaith, H.J.; Herz, L.M. High charge carrier mobilities and lifetimes in organolead trihalide perovskites. Adv. Mater. 2014, 26, 1584-1589. [CrossRef] [PubMed]

40. Wang, H.; Kim, D.H. Perovskite-based photodetectors: Materials and devices. Chem. Soc. Rev. 2017, 46, 5204-5236. [CrossRef] [PubMed]

41. Leyden, M.R.; Meng, L.Q.; Jiang, Y.; Ono, L.K.; Qiu, L.B.; Juarez-Perez, E.J.; Qin, C.J.; Adachi, C.; Qi, Y.B. Methylammonium lead bromide perovskite light emitting diodes by chemical vapor deposition. J. Phys. Chem. Lett. 2017, 8, 3193-3198. [CrossRef] [PubMed]

42. Zhu, H.; Fu, Y.; Meng, F.; Wu, X.; Gong, Z.; Ding, Q.; Gustafsson, M.V.; Trinh, M.T.; Jin, S.; Zhu, X.-Y. Lead halide perovskite nanowire lasers with low lasing thresholds and high quality factors. Nat. Mater. 2015, 14, 636-642. [CrossRef]

43. Ward, J.W.; Smith, H.L.; Zeidell, A.; Diemer, P.J.; Baker, S.R.; Lee, H.; Payne, M.M.; Anthony, J.E.; Guthold, M.; Jurchescu, O.D. Solution-processed organic and halide perovskite transistors on hydrophobic surfaces. ACS Appl. Mater. Interfaces 2017, 9, 18120-18126. [CrossRef] [PubMed]

44. Jella, V.; Ippili, S.; Eom, J.-H.; Pammi, S.V.N.; Jung, J.-S.; Tran, V.-D.; Nguyen, V.H.; Kirakosyan, A.; Yun, S.; Kim, D.; et al. A comprehensive review of flexible piezoelectric generators based on organic-inorganic metal halide perovskites. Nano Energy 2019, 57, 74-93. [CrossRef]

45. Ippili, S.; Jella, V.; Kim, J.; Hong, S.; Yoon, S.-G. Unveiling predominant air-stable organotin bromide perovskite toward mechanical energy harvesting. ACS Appl. Mater. Interfaces 2020, 12, 16469-16480. [CrossRef] [PubMed]

46. Ding, R.; Zhang, X.; Chen, G.; Wang, H.; Kishor, R.; Xiao, J.; Gao, F.; Zeng, K.; Chen, X.; Sun, X.W.; et al. High-performance piezoelectric nanogenerators composed of formamidinium lead halide perovskite nanoparticles and poly(vinylidene fluoride). Nano Energy 2017, 37, 126-135. [CrossRef]

47. Dhar, J.; Sil, S.; Hoque, N.A.; Dey, A.; Das, S.; Ray, P.P.; Sanyal, D. Lattice-defect-induced piezo response in methylammoniumlead-iodide perovskite based nanogenerator. ChemistrySelect 2018, 3, 5304-5312. [CrossRef]

48. Sultana, A.; Ghosh, S.K.; Alam, M.M.; Sadhukhan, P.; Roy, K.; Xie, M.; Bowen, C.R.; Sarkar, S.; Das, S.; Middya, T.R.; et al. Methylammonium lead iodide incorporated poly(vinylidene fluoride) nanofibers for flexible piezoelectric-pyroelectric nanogenerator. ACS Appl. Mater. Interfaces 2019, 11, 27279-27287. [CrossRef]

49. Kim, W.-G.; Kim, D.-W.; Tcho, I.-W.; Kim, J.-K.; Kim, M.-S.; Choi, Y.-K. Triboelectric nanogenerator: Structure, mechanism, and applications. ACS Nano 2021, 15, 258-287. [CrossRef] [PubMed]

50. Sultana, A.; Alam, M.M.; Sadhukhan, P.; Ghorai, U.K.; Das, S.; Middya, T.R.; Mandal, D. Organo-lead halide perovskite regulated green light emitting poly(vinylidene fluoride) electrospun nanofiber mat and its potential utility for ambient mechanical energy harvesting application. Nano Energy 2018, 49, 380-392. [CrossRef]

51. Ippili, S.; Jella, V.; Eom, S.; Hong, S.; Yoon, S.-G. Light-driven piezo- and triboelectricity in organic-inorganic metal trihalide perovskite toward mechanical energy harvesting and self-powered sensor application. ACS Appl. Mater. Interfaces 2020, 12, 50472-50483. [CrossRef] [PubMed]

52. Su, L.; Zhao, Z.; Li, H.; Wang, Y.; Kuang, S.; Cao, G.; Wang, Z.; Zhu, G. Photoinduced enhancement of a triboelectric nanogenerator based on an organolead halide perovskite. J. Mater. Chem. C 2016, 4, 10395-10399. [CrossRef]

53. Xu, Z.; Wu, C.; Zhu, Y.; Ju, S.; Ma, F.; Guo, T.; Li, F.; Kim, T.W. Bio-inspired smart electronic-skin based on inorganic perovskite nanoplates for application in photomemories and mechanoreceptors. Nanoscale 2021, 13, 253-260. [CrossRef] [PubMed] 
54. Yang, X.D.; Han, J.J.; Wang, G.; Liao, L.P.; Xu, C.Y.; Hu, W.; Li, P.; Wu, B.; Elseman, A.M.; Zhou, G.D.; et al. Robust perovskite-based triboelectric nanogenerator enhanced by broadband light and interface engineering. J. Mater. Sci. 2019, 54, 9004-9016. [CrossRef]

55. Eom, J.H.; Choi, H.J.; Pammi, S.V.N.; Tran, V.D.; Kim, Y.J.; Kim, H.J.; Yoon, S.-G. Self-powered pressure and light sensitive bimodal sensors based on long-term stable piezo-photoelectric $\mathrm{MAPbI}_{3}$ thin films. J. Mater. Chem. C 2018, 6, 2786-2792. [CrossRef]

56. Goldschmidt, V.M. Krystallbau und chemische Zusammensetzung. Ber. Dtsch. Chem. Ges. 1927, 60, 1263-1296. [CrossRef]

57. Kieslich, G.; Sun, S.; Cheetham, A.K. Solid-state principles applied to organic-inorganic perovskites: New tricks for an old dog. Chem. Sci. 2014, 5, 4712-4715. [CrossRef]

58. Park, N.-G. Perovskite solar cells: An emerging photovoltaic technology. Mater. Today 2015, 18, 65-72. [CrossRef]

59. Assadi, M.K.; Bakhoda, S.; Saidur, R.; Hanaei, H. Recent progress in perovskite solar cells. Renew. Sustain. Energy Rev. 2017, 81, 2812-2822. [CrossRef]

60. Li, Z.; Yang, M.; Park, J.-S.; Wei, S.-H.; Berry, J.; Zhu, K. Stabilizing perovskite structures by tuning tolerance factor: Formation of formamidinium and cesium lead iodide solid-state alloys. Chem. Mater. 2016, 28, 284-292. [CrossRef]

61. Wang, M.; Feng, Y.; Bian, J.; Liu, H.; Shi, Y. A comparative study of one-step and two-step approaches for $\mathrm{MAPbI}_{3}$ perovskite layer and its influence on the performance of mesoscopic perovskite solar cell. Chem. Phys. Lett. 2018, 692, 44-49. [CrossRef]

62. Kirakosyan, A.; Kim, J.; Lee, S.W.; Swathi, I.; Yoon, S.-G.; Choi, J. Optical properties of colloidal $\mathrm{CH}_{3} \mathrm{NH}_{3} \mathrm{PbBr}_{3}$ nanocrystals by controlled growth of lateral dimension. Cryst. Growth Des. 2017, 17, 794-799. [CrossRef]

63. Liu, M.; Johnston, M.B.; Snaith, H.J. Efficient planar heterojunction perovskite solar cells by vapour deposition. Nature 2013, 501, 395-398. [CrossRef] [PubMed]

64. Chen, Q.; Zhou, H.; Hong, Z.; Luo, S.; Duan, H.-S.; Wang, H.-H.; Liu, Y.; Li, G.; Yang, Y. Planar heterojunction perovskite solar cells via vapor-assisted solution process. J. Am. Chem. Soc. 2014, 136, 622-625. [CrossRef] [PubMed]

65. Ippili, S.; Jella, V.; Kim, J.; Hong, S.; Yoon, S.-G. Enhanced piezoelectric output performance via control of dielectrics in $\mathrm{Fe} 2+$-incorporated $\mathrm{MAPbI}_{3}$ perovskite thin films: Flexible piezoelectric generators. Nano Energy 2018, 49, 247-256. [CrossRef]

66. Jella, V.; Ippili, S.; Yoon, S.-G. Halide $(\mathrm{Cl} / \mathrm{Br})$-incorporated organic-inorganic metal trihalide perovskite films: Study and investigation of dielectric properties and mechanical energy harvesting performance. ACS Appl. Electron. Mater. 2020, 2, 2579-2590. [CrossRef]

67. Park, H.; Ha, C.; Lee, J.-H. Advances in piezoelectric halide perovskites for energy harvesting applications. J. Mater. Chem. A 2020, 8, 24353-24367. [CrossRef]

68. Jella, V.; Ippili, S.; Eom, J.-H.; Choi, J.; Yoon, S.-G. Enhanced output performance of a flexible piezoelectric energy harvester based on stable $\mathrm{MAPbI}_{3}-\mathrm{PVDF}$ composite films. Nano Energy 2018, 53, 46-56. [CrossRef]

69. Kutes, Y.; Ye, L.; Zhou, Y.; Pang, S.; Huey, B.D.; Padture, N.P. Direct observation of ferroelectric domains in solution-processed $\mathrm{CH}_{3} \mathrm{NH}_{3} \mathrm{PbI}_{3}$ perovskite thin films. J. Phys. Chem. Lett. 2014, 5, 3335-3339. [CrossRef] [PubMed]

70. Dong, Q.; Song, J.; Fang, Y.; Shao, Y.; Ducharme, S.; Huang, J. Lateral-structure single-crystal hybrid perovskite solar cells via piezoelectric poling. Adv. Mater. 2016, 28, 2816-2821. [CrossRef]

71. Kim, Y.J.; Dang, T.V.; Choi, H.J.; Park, B.J.; Eom, J.H.; Song, H.A.; Seol, D.; Kim, Y.; Shin, S.H.; Nah, H.; et al. Piezoelectric properties of $\mathrm{CH}_{3} \mathrm{NH}_{3} \mathrm{PbI}_{3}$ perovskite thin films and their applications in piezoelectric generators. J. Mater. Chem. A 2016, 4, 756-763. [CrossRef]

72. Ippili, S.; Jella, V.; Thomas, A.M.; Yoon, C.; Jung, J.-S.; Yoon, S.-G. ZnAl-LDH-induced electroactive $\beta$-phase and controlled dielectrics of PVDF for a high-performance triboelectric nanogenerator for humidity and pressure sensing applications. J. Mater. Chem. A 2021. [CrossRef]

73. Yang, T.Y.; Gregori, G.; Pellet, N.; Grätzel, M.; Maier, J. The significance of ion conduction in a hybrid organic-inorganic lead-iodide-based perovskite photosensitizer. Angew. Chem. Int. Ed. 2015, 54, 7905-7910. [CrossRef] [PubMed]

74. Liu, Y.; Sun, J.; Yang, Z.; Yang, D.; Ren, X.; Xu, H.; Yang, Z.; Liu, S.F. 20-mm-large single-crystalline formamidinium-perovskite wafer for mass production of integrated photodetectors. Adv. Opt. Mater. 2016, 4, 1829-1837. [CrossRef]

75. Juarez-Perez, E.J.; Sanchez, R.S.; Badia, L.; Garcia-Belmonte, G.; Kang, Y.S.; Mora-Sero, I.; Bisquert, J. Photoinduced giant dielectric constant in lead halide perovskite solar cells. J. Phys. Chem. Lett. 2014, 5, 2390-2394. [CrossRef]

76. Almond, D.P.; Bowen, C.R. An explanation of the photoinduced giant dielectric constant of lead halide perovskite solar cells. J. Phys. Chem. Lett. 2015, 6, 1736-1740. [CrossRef]

77. Song, J.; Xiao, Z.; Chen, B.; Prockish, S.; Chen, X.; Rajapitamahuni, A.; Zhang, L.; Huang, J.; Hong, X. Enhanced piezoelectric response in hybrid lead halide perovskite thin films via interfacing with ferroelectric $\mathrm{PbZr}_{0.2} \mathrm{Ti}_{0.8} \mathrm{O}_{3}$. ACS Appl. Mater. Interfaces 2018, 10, 19218-19225. [CrossRef]

78. Ding, R.; Liu, H.; Zhang, X.; Xiao, J.; Kishor, R.; Sun, H.; Zhu, B.; Chen, G.; Gao, F.; Feng, X.; et al. Flexible piezoelectric nanocomposite generators based on formamidinium lead halide perovskite nanoparticles. Adv. Funct. Mater. 2016, 26, 7708-7716. [CrossRef]

79. Kim, D.B.; Park, K.H.; Cho, Y.S. Origin of high piezoelectricity of inorganic halide perovskite thin films and their electromechanical energy-harvesting and physiological current-sensing characteristics. Energy Environ. Sci. 2020, 13, 2077-2086. [CrossRef]

80. Su, L.; Zhao, Z.X.; Li, H.Y.; Yuan, J.; Wang, Z.L.; Cao, G.Z.; Zhu, G. High-performance organolead halide perovskite-based self-powered triboelectric photodetector. ACS Nano 2015, 9, 11310-11316. [CrossRef]

81. Wang, Y.; Duan, J.; Yang, X.; Liu, L.; Zhao, L.; Tang, Q. The unique dielectricity of inorganic perovskites toward high-performance triboelectric nanogenerators. Nano Energy 2020, 69, 104418. [CrossRef] 
82. Lou, M.; Abdalla, I.; Zhu, M.; Wei, X.; Yu, J.; Li, Z.; Ding, B. Highly wearable, breathable and washable sensing textile for human motion and pulse monitoring. ACS Appl. Mater. Interfaces 2020, 12, 1597-1605. [CrossRef]

83. Yuan, H.; Lei, T.; Qin, Y.; Yang, R. Flexible electronic skins based on piezoelectric nanogenerators and piezotronics. Nano Energy 2019, 59, 84-90. [CrossRef]

84. Shi, M.; Holmes, A.S.; Yeatman, E.M. Piezoelectric wind velocity sensor based on the variation of galloping frequency with drag force. Appl. Phys. Lett. 2020, 116, 264101. [CrossRef]

85. Zhong, J.; Ma, Y.; Song, Y.; Zhong, Q.; Chu, Y.; Karakurt, I.; Bogy, D.B.; Lin, L. A flexible piezoelectret actuator/sensor patch for mechanical human-machine interfaces. ACS Nano 2019, 13, 7107-7116. [CrossRef] [PubMed]

86. Wang, Y.; Yang, X.; Yu, X.; Duan, J.; Yang, Q.; Duan, Y.; Tang, Q. Triboelectric charging behaviors and photoinduced enhancement of alkaline earth ions doped inorganic perovskite triboelectric nanogenerators. Nano Energy 2020, 77, 105280. [CrossRef]

87. Fan, F.-R.; Tian, Z.-Q.; Wang, Z.L. Flexible triboelectric generator. Nano Energy 2012, 1, 328-334. [CrossRef]

88. Jin, L.; Tao, J.; Bao, R.; Sun, L.; Pan, C. Self-powered real-time movement monitoring sensor using triboelectric nanogenerator technology. Sci. Rep. 2017, 7, 10521. [CrossRef] [PubMed]

89. Lai, S.-N.; Chang, C.-K.; Yang, C.-S.; Su, C.-W.; Leu, C.-M.; Chu, Y.-H.; Sha, P.-W.; Wu, J.M. Ultrasensitivity of self-powered wireless triboelectric vibration sensor for operating in underwater environment based on surface functionalization of rice husks. Nano Energy 2019, 60, 715-723. [CrossRef]

90. Zhou, Q.; Park, J.G.; Kim, K.N.; Thokchom, A.K.; Bae, J.; Baik, J.M.; Kim, T. Transparent-flexible-multimodal triboelectric nanogenerators for mechanical energy harvesting and self-powered sensor applications. Nano Energy 2018, 48, 471-480. [CrossRef]

91. Bowen, C.R.; Taylor, J.; LeBoulbar, E.; Zabek, D.; Chauhan, A.; Vaish, R. Pyroelectric materials and devices for energy harvesting applications. Energy Environ. Sci. 2014, 7, 3836-3856. [CrossRef]

92. Leng, Q.; Chen, L.; Guo, H.; Liu, J.; Liu, G.; Hu, C.; Xi, Y. Harvesting heat energy from hot/cold water with a pyroelectric generator. J. Mater. Chem. A 2014, 2, 11940-11947. [CrossRef]

93. Xue, H.; Yang, Q.; Wang, D.; Luo, W.; Wang, W.; Lin, M.; Liang, D.; Luo, Q. A wearable pyroelectric nanogenerator and self-powered breathing sensor. Nano Energy 2017, 38, 147-154. [CrossRef]

94. Wang, X.D.; Wolfbeis, O.S.; Meier, R.J. Luminescent probes and sensors for temperature. Chem. Soc. Rev. 2013, 42, 7834-7869. [CrossRef]

95. Lian, X.; Zhao, D.; Cui, Y.; Yang, Y.; Qian, G. A near infrared luminescent metal-organic framework for temperature sensing in the physiological range. Chem. Commun. 2015, 51, 17676-17679. [CrossRef]

96. Kong, W.; Ye, Z.; Qi, Z.; Zhang, B.; Wang, M.; Rahimi-Iman, A.; Wu, H. Characterization of an abnormal photoluminescence behavior upon crystal-phase transition of perovskite $\mathrm{CH}_{3} \mathrm{NH}_{3} \mathrm{PbI}_{3}$. Phys. Chem. Chem. Phys. 2015, 17, 16405-16411. [CrossRef]

97. Xing, J.; Liu, X.F.; Zhang, Q.; Ha, S.T.; Yuan, Y.W.; Shen, C.; Sum, T.C.; Xiong, Q. Vapor phase synthesis of organometal halide perovskite nanowires for tunable room-temperature nanolasers. Nano Lett. 2015, 15, 4571-4577. [CrossRef] [PubMed]

98. Zhu, Z.; Sun, Q.; Zhang, Z.; Dai, J.; Xing, G.; Li, S.; Huang, X.; Huang, W. Metal halide perovskites: Stability and sensing-ability. J. Mater. Chem. C 2018, 6, 10121-10137. [CrossRef]

99. Ippili, S.; Jella, V.; Eom, J.-H.; Kim, J.; Hong, S.; Choi, J.-S.; Tran, V.-D.; Van Hieu, N.; Kim, Y.-J.; Kim, H.-J.; et al. An ecofriendly flexible piezoelectric energy harvester that delivers high output performance is based on lead-free MASnI films and MASnI $_{3}$ PVDF composite films. Nano Energy 2019, 57, 911-923. [CrossRef]

100. Chortos, A.; Bao, Z.A. Skin-inspired electronic devices. Mater. Today 2014, 17, 321-331. [CrossRef]

101. Chen, X.; Li, X.; Shao, J.; An, N.; Tian, H.; Wang, C.; Han, T.; Wang, L.; Lu, B. High-performance piezoelectric nanogenerators with imprinted $\mathrm{P}(\mathrm{VDF}-\mathrm{TrFE}) / \mathrm{BaTiO}_{3}$ nanocomposite micropillars for self-powered flexible sensors. Small 2017, 13,1604245 . [CrossRef] [PubMed]

102. Chen, X.; Shao, J.; An, N.; Li, X.; Tian, H.; Xu, C.; Ding, Y. Self-Powered flexible pressure sensors with vertically well-aligned piezoelectric nanowire arrays for monitoring vital signs. J. Mater. Chem. C 2015, 3, 11806-11814. [CrossRef]

103. Chen, X.; Parida, K.; Wang, J.; Xiong, J.; Lin, M.-F.; Shao, J.; Lee, P.S. A stretchable and transparent nanocomposite nanogenerator for self-powered physiological monitoring. ACS Appl. Mater. Interfaces 2017, 9, 42200-42209. [CrossRef] [PubMed]

104. Fan, F.R.; Tang, W.; Wang, Z.L. Flexible nanogenerators for energy harvesting and self-powered electronics. Adv. Mater. 2016, 28, 4283-4305. [CrossRef]

105. Chun, S.; Son, W.; Kim, H.; Lim, S.K.; Pang, C.; Choi, C. Self-powered pressure- and vibration-sensitive tactile sensors for learning technique-based neural finger skin. Nano Lett. 2019, 19, 3305-33129. [CrossRef]

106. Zhao, G.; Zhang, Y.; Shi, N.; Liu, Z.; Zhang, X.; Wu, M.; Pan, C.; Liu, H.; Li, L.; Wang, Z.L. Transparent and stretchable triboelectric nanogenerator for self-powered tactile sensing. Nano Energy 2019, 59, 302-310. [CrossRef]

107. Parida, K.; Xiong, J.Q.; Zhou, X.R.; Lee, P.S. Progress on triboelectric nanogenerator with stretchability, self-healability and bio-compatibility. Nano Energy 2019, 59, 237-257. [CrossRef]

108. Bhavanasi, V.; Kumar, V.; Parida, K.; Wang, J.X.; Lee, P.S. Enhanced piezoelectric energy harvesting performance of flexible pvdf-trfe bilayer films with graphene oxide. ACS Appl. Mater. Interfaces 2016, 8, 521-529. [CrossRef]

109. Parida, K.; Kumar, V.; Jiangxin, W.; Bhavanasi, V.; Bendi, R.; Lee, P.S. Highly transparent, stretchable, and self-healing ionic-skin triboelectric nanogenerators for energy harvesting and touch applications. Adv. Mater. 2017, 29, 1702181. [CrossRef]

110. Xiong, J.; Cui, P.; Chen, X.; Wang, J.; Parida, K.; Lin, M.F.; Lee, P.S. Skin-touch-actuated textile-based triboelectric nanogenerator with black phosphorus for durable biomechanical energy harvesting. Nat. Commun. 2018, 9, 4280. [CrossRef] 
111. Parida, K.; Thangavel, G.; Cai, G.; Zhou, X.; Park, S.; Xiong, J.; Lee, P.S. Extremely stretchable and self-healing conductor based on thermoplastic elastomer for all-three-dimensional printed triboelectric nanogenerator. Nat. Commun. 2019, 10, 2158. [CrossRef] [PubMed]

112. Vallem, V.; Sargolzaeiaval, Y.; Ozturk, M.; Lai, Y.-C.; Dickey, M.D. Energy harvesting and storage with soft and stretchable materials. Adv. Mater. 2021, 33, 2004832. [CrossRef] [PubMed]

113. Lai, Y.-C.; Deng, J.; Liu, R.; Hsiao, Y.-C.; Zhang, S.L.; Peng, W.; Wu, H.-M.; Wang, X.; Wang, Z.L. Actively perceiving and responsive soft robots enabled by self-powered, highly extensible, and highly sensitive triboelectric proximity- and pressure-sensing skins. Adv. Mater. 2018, 30, 1801114. [CrossRef] [PubMed]

114. Lai, Y.-C.; Deng, J.; Niu, S.; Peng, W.; Wu, C.; Liu, R.; Wen, Z.; Wang, Z.L. Electric eel-skin-inspired mechanically durable and super-stretchable nanogenerator for deformable power source and fully autonomous conformable electronic-skin applications. Adv. Mater. 2016, 28, 10024-10032. [CrossRef] [PubMed]

115. Seo, J.; Park, S.; Kim, Y.C.; Jeon, N.J.; Noh, J.H.; Yoon, S.C.; Seok, S.I. Benefits of very thin PCBM and LiF layers for solutionprocessed p-i-n perovskite solar cells. Energy Environ. Sci. 2014, 7, 2642-2646. [CrossRef]

116. Liu, S.; Zheng, F.; Grinberg, I.; Rappe, A.M. Photoferroelectric and photopiezoelectric properties of organometal halide perovskites. J. Phys. Chem. Lett. 2016, 7, 1460-1465. [CrossRef]

117. Deswal, S.; Singh, S.K.; Rambabu, P.; Kulkarni, P.; Vaitheeswaran, G.; Praveenkumar, B.; Ogale, S.; Boomishankar, R. Flexible composite energy harvesters from ferroelectric $\mathrm{A}_{2} \mathrm{MX}_{4}$-type hybrid halogenometallates. Chem. Mater. 2019, 31, 4545-4552. [CrossRef]

118. Jella, V.; Ippili, S.; Eom, J.H.; Kim, Y.J.; Kim, H.J.; Yoo, S.-G. A novel approach to ambient energy (thermoelectric, piezoelectric and solar-TPS) harvesting: Realization of a single structured TPS-fusion energy device using $\mathrm{MAPbI}_{3}$. Nano Energy 2018, 52, 11-21. [CrossRef]

119. Pandey, R.; Sb, G.; Grover, S.; Singh, S.K.; Kadam, A.; Ogale, S.; Waghmare, U.V.; Rao, V.R.; Kabra, D. Microscopic origin of piezoelectricity in lead-free halide perovskite: Application in nanogenerator design. ACS Energy Lett. 2019, 4, 1004-1011. [CrossRef]

120. Huang, G.; Khan, A.A.; Rana, M.M.; Xu, C.; Xu, S.; Saritas, R.; Zhang, S.; Rahmand, E.; Turban, P.; Girard, S.; et al. Achieving ultrahigh piezoelectricity in organic-inorganic vacancy-ordered halide double perovskites for mechanical energy harvesting. ACS Energy Lett. 2021, 6, 16-23. [CrossRef]

121. Guan, H.; Lv, D.; Zhong, T.; Dai, Y.; Xing, L.; Xue, X.; Zhnag, Y.; Zhan, Y. Self-powered, wireless-control, neural-stimulating electronic skin for in vivo characterization of synaptic plasticity. Nano Energy 2020, 67, 104182. [CrossRef]

122. Bao, C.; Yang, J.; Bai, S.; Xu, W.; Yan, Z.; Xu, Q.; Liu, J.; Zhang, W.; Gao, F. High performance and stable all-inorganic metal halide perovskite-based photodetectors for optical communication applications. Adv. Mater. 2018, 30, 1803422. [CrossRef] [PubMed]

123. Chouhan, L.; Ghimire, S.; Subrahmanyam, C.; Miyasaka, T.; Biju, V. Synthesis, optoelectronic properties and applications of halide perovskites. Chem. Soc. Rev. 2020, 49, 2869-2885. [CrossRef]

124. Manser, J.S.; Christians, J.A.; Kamat, P.V. Intriguing optoelectronic properties of metal halide perovskites. Chem. Rev. 2016, 116, 12956-13008. [CrossRef]

125. Liu, C.-K.; Tai, Q.; Wang, N.; Tang, G.; Loi, H.-L.; Yan, F. Sn-based perovskite for highly sensitive photodetectors. Adv. Sci. 2019, 6, 1900751. [CrossRef]

126. Li, Y.; Shi, Z.; Liang, W.; Wang, L.; Li, S.; Zhang, F.; Ma, Z.; Wang, Y.; Tian, Y.; Wu, D.; et al. Highly stable and spectrum-selective ultraviolet photodetectors based on lead-free copper-based perovskites. Mater. Horiz. 2020, 7, 530-540. [CrossRef]

127. Hsiao, V.K.S.; Leung, S.-F.; Hsiao, Y.-C.; Kung, P.-K.; Lai, Y.-C.; Lin, Z.-H.; Salama, K.N.; Alshareef, H.N.; Wang, Z.L.; He, J.-H. Photo-carrier extraction by triboelectricity for carrier transport layer-free photodetectors. Nano Energy 2019, 65, 103958-103966. [CrossRef]

128. Liang, W.; Shi, Z.; Li, Y.; Ma, J.; Yin, S.; Chen, X.; Wu, D.; Tian, Y.; Tian, Y.; Zhang, Y.; et al. Strategy of all-inorganic Cs3Cu2I5/SiCore/Shell nanowire heterojunction for stable and ultraviolet-enhanced broadband photodetectors with imaging capability. ACS Appl. Mater. Interfaces 2020, 12, 37363-37374. [CrossRef]

129. Luo, J.; Li, S.; Wu, H.; Zhou, Y.; Li, Y.; Liu, J.; Li, J.; Li, K.; Yi, F.; Niu, G.; et al. $\mathrm{Cs}_{2} \mathrm{AgInCl}_{6}$ double perovskite single crystals: Parity forbidden transitions and their application for sensitive and fast uv photodetectors. ACS Photonics 2018, 5, 398-405. [CrossRef]

130. Yang, Y.; Dai, H.; Yang, F.; Zhang, Y.; Luo, D.; Zhang, X.; Wang, K.; Sun, X.W.; Yao, J. All-Perovskite photodetector with fast response. Nanoscale Res. Lett. 2019, 14, 291. [CrossRef] [PubMed]

131. Sultana, A.; Sadhukhan, P.; Alam, M.M.; Das, S.; Middya, T.R.; Mandal, D. Organo-lead halide perovskite induced electroactive $\beta$-phase in porous pvdf films: An excellent material for photoactive piezoelectric energy harvester and photodetector. ACS Appl. Mater. Interfaces 2018, 10, 4121-4130. [CrossRef]

132. Si, S.K.; Paria, S.; Karan, S.K.; Ojha, S.; Das, A.K.; Maitra, A.; Bera, A.; Halder, L.; De, A.; Khatua, B.B. In situ grown organo-lead bromide perovskite induced electroactive $\gamma$-phase in aerogel PVDF film: An efficient photoactive material for piezoelectric energy harvesting and photodetector application. Nanoscale 2020, 12, 7214-7230. [CrossRef]

133. Huang, C.-B.; Witomska, S.; Aliprandi, A.; Stoeckel, M.-A.; Bonini, M.; Ciesielski, A.; Samorì, P. Molecule-Graphene hybrid materials with tunable mechanoresponse: Highly sensitive pressure sensors for health monitoring. Adv. Mater. 2019, 31, 1804600. [CrossRef] 
134. Shekhawat, G.S.; Ramachandran, S.; Sharahi, H.J.; Sarkar, S.; Hujsak, K.; Li, Y.; Hagglund, K.; Kim, S.; Aden, G.; Chand, A.; et al. Micromachined chip scale thermal sensor for thermal imaging. ACS Nano 2018, 12, 1760-1767. [CrossRef]

135. Liu, F.; Zheng, S.; He, X.; Chaturvedi, A.; He, J.; Chow, W.L.; Mion, T.R.; Wang, X.; Zhou, J.; Fu, Q.; et al. Highly sensitive detection of polarized light using anisotropic $2 \mathrm{D} \mathrm{ReS}_{2}$. Adv. Funct. Mater. 2016, 26, 1169-1177. [CrossRef]

136. Chang, S.-P.; Chang, S.-J.; Lu, C.-Y.; Li, M.-J.; Hsu, C.-L.; Chiou, Y.-Z.; Hsueh, T.-J.; Chen, I.-C. A ZnO nanowire-based humidity sensor. Superlattices Microstruct. 2010, 47, 772-778. [CrossRef]

137. Kanao, K.; Harada, S.; Yamamoto, Y.; Honda, W.; Arie, T.; Akita, S.; Takei, K. Highly selective flexible tactile strain and temperature sensors against substrate bending for an artificial skin. RSC Adv. 2015, 5, 30170-30174. [CrossRef]

138. Engel, J.; Chen, J.; Fan, Z.; Liu, C. Polymer micromachined multimodal tactile sensors. Sens. Actuators A 2005, 117, 50-61. [CrossRef]

139. Röhm, H.; Leonhard, T.; Hoffmann, M.J.; Colsmann, A. Ferroelectric domains in methylammonium lead iodide perovskite thin-films. Energy Environ. Sci. 2017, 10, 950-955. [CrossRef]

140. Rakita, Y.; B-Elli, O.; Meirzadeh, E.; Kaslasi, H.; Pelea, Y.; Hodes, G.; Lubomirsky, I.; Oron, D.; Ehre, D.; Cahen, D. Tetragonal $\mathrm{CH}_{3} \mathrm{NH}_{3} \mathrm{PbI}_{3}$ is ferroelectric. Proc. Natl. Acad. Sci. USA 2017, 114, E5504-E5512. [CrossRef] [PubMed]

141. He, Y.; Galli, G. Perovskites for solar thermoelectric applications: A first principle study of $\mathrm{CH}_{3} \mathrm{NH}_{3} \mathrm{AI}_{3}(\mathrm{~A}=\mathrm{Pb}$ and Sn). Chem. Mater. 2014, 26, 5394-5400. [CrossRef]

142. Ma, C.; Lo, M.F.; Lee, C.S. Stabilization of organometallic halide perovskite nanocrystals in aqueous solutions and their applications in copper ion detection. Chem. Commun. 2018, 54, 5784-5787. [CrossRef] [PubMed]

143. Sasmal, S.; Sinha, A.; Donnadieu, B.; Pala, R.G.S.; Sivakumar, S.; Valiyaveettil, S. Volatility and chain length interplay of primary amines: Mechanistic investigation on the stability and reversibility of ammonia-responsive hybrid perovskites. ACS Appl. Mater. Interfaces 2018, 10, 6711-6718. [CrossRef] [PubMed] 